\title{
ENDOMORPHISMS OF VERMA MODULES FOR RATIONAL CHEREDNIK ALGEBRAS
}

\author{
G. BELLAMY* \\ School of \\ Mathematics and Statistics \\ University of Glasgow \\ University Gardens \\ Glasgow G12 8QW, Scotland \\ gwyn.bellamy@glasgow.ac.uk
}

\begin{abstract}
We study the endomorphism algebras of Verma modules for rational Cherednik algebras at $t=0$. It is shown that, in many cases, these endomorphism algebras are quotients of the centre of the rational Cherednik algebra. Geometrically, they define Lagrangian subvarieties of the generalized Calogero-Moser space. In the introduction, we motivate our results by describing them in the context of derived intersections of Lagrangians.
\end{abstract}

\section{Introduction}

The quest for a direct bridge between the geometric world of Fukaya categories and the world of (algebraic or analytic) microlocal sheaves on a symplectic manifold is ongoing; it is currently the subject of intense research. Though considerable progress has recently been made, for instance with Tamarkin's seminal work on quantizations of Lagrangians [32], it seems that concrete, computable examples of this expected correspondence are still desirable to aid one's (or at least the author's) intuition. One abode where such computable, though non-trivial, examples live is that of rational Cherednik algebras, beginning for instance with the results of [29]. The corresponding symplectic manifold is the generalized Calogero-Moser space, or often times, more appropriately, a symplectic resolution of this space.

Though we have no idea what the appropriate definition of Fukaya category should be in this case, or its possible relation to microlocal sheaves on the generalized Calogero-Moser space $X$, we study in this article a shadow of such a hoped for relationship. For argument's sake, we take the objects of the Fukaya category, a 2-category, to be Lagrangian (or more generally coisotropic) subvarieties and the hom spaces given by derived intersections. Then, in the shadow of the Fukaya category, the derived intersections are replaced by the Tor and Ext groups between the structure sheaves of the Lagrangians subvarieties, i.e., by the (co)homology

DOI: $10.1007 / \mathrm{s} 00031-014-9281-\mathrm{x}$

* The author would like to thank the referees for carefully reading the article. The author is supported by the EPSRC grant EP-H028153.

Received October 20, 2012. Accepted May 20, 2014.

Published online June 15, 2014.

Corresponding Author: G. Bellamy, e-mail: gwyn.bellamy@glasgow.ac.uk. 
of the derived intersections. On the microlocal side, we consider not quantized sheaves on the space $X$, but those sheaves of $\mathcal{O}_{X}$-modules that are quantizable, i.e., that admit some quantization. It is these Tor and Ext groups that we study in this article.

One major advantage of working on the generalized Calogero-Moser space, even though it is a singular symplectic variety, is that it is equipped with a canonical quantization by virtue of the fact that it is the centre of the rational Cherednik algebra.

Our original motivation for the current work was quite different - see the end of the introduction.

\section{Quantizable modules}

Associated to each complex reflection group $(\mathfrak{h}, W)$, are the rational Cherednik algebras, a family of algebras depending on a pair of parameters $(t, \mathbf{c})$, where $t \in \mathbb{C}$. When $t=0$, the algebra $\mathrm{H}_{\mathbf{c}}$ is a finite module over its centre $\mathbf{Z}_{\mathbf{c}}$. The algebra $Z_{\mathbf{c}}$ is the coordinate ring of a symplectic variety $X_{\mathbf{c}}$, and the geometry of this symplectic variety is intimately entwined with the representation theory of $\mathrm{H}_{\mathbf{c}}$. When $t \neq 0$, the rational Cherednik algebra is strongly non-commutative and provides a canonical quantization of the symplectic variety $X_{\mathbf{c}}$. For fixed $\mathbf{c}$, one can also consider the $\mathbb{C}[\mathbf{t}]$-algebra $\mathrm{H}_{\mathbf{t}, \mathbf{c}}$, which is flat over $\mathbf{t}$.

$\mathrm{A} \mathrm{H}_{\mathbf{c}}$-module $M$ is said to be quantizable if it can be extended to a $\mathrm{H}_{\mathbf{t}, \mathbf{c}}$-module, flat over $\mathbb{C}[\mathbf{t}]$. In fact, for what follows, the existence of an extension to the 3rd infinitesimal neighborhood of zero in Spec $\mathbb{C}[\mathbf{t}]$ suffices. Gabber's Theorem implies that the support $\operatorname{Supp} M \subset X_{\mathbf{c}}$ of a quantizable module $M$ is coisotropic, i.e., is defined by the vanishing of an involutive ideal.

Let $M, N$ be quantizable left $\mathrm{H}_{\mathbf{c}}$-modules and $M^{\prime}$ a quantizable right $\mathrm{H}_{\mathbf{c}}$-module. Then, by the construction described in section 3.1 of [2], the graded vector spaces $\operatorname{Ext}_{\mathrm{H}_{\mathbf{c}}}^{\bullet}(M, N)$ and $\operatorname{Tor}_{\bullet}^{\mathrm{H}_{\mathbf{c}}}\left(M^{\prime}, N\right)$ carry a canonical differential, the virtual de Rham differential, making each into a complex. The cohomology of $\operatorname{Ext}_{\mathrm{H}_{\mathrm{c}}}(M, N)$, resp. $\operatorname{Tor}_{\bullet}^{\mathrm{H}_{\mathrm{c}}}\left(M^{\prime}, N\right)$, is commonly called the virtual de Rham cohomology of the pair $(M, N)$, resp. the virtual de Rham homology of $\left(M^{\prime}, N\right)$. In the context of symplectic geometry, virtual de Rham (co)homology plays a (conjectural) role in counting intersection numbers of Lagrangian intersections; see [3]. With regards to the possible relationship between virtual de Rham (co)homology and microlocal sheaves; see also [30, Rem. 7.7].

A pair of (left or right) quantizable modules $M$ and $N$ are said to have smooth intersection if $\operatorname{Supp} M \cap \operatorname{Supp} N$ is contained in the smooth locus of $X_{\mathbf{c}}$. Moreover, $M$ is said to be simple if $e M$ is a cyclic $\mathbf{Z}_{\mathbf{c}}$-module; the terminology comes from the representation theory of deformation-quantization algebras. In this case, results of [2], together with some basic Mortia theory imply:

Proposition 1. Assume that $M$ and $N$ are simple, quantizable left $\mathrm{H}_{\mathbf{c}}$-modules, with smooth intersection and $M^{\prime}$ a quantizable right $\mathrm{H}_{\mathbf{c}}$-module such that $\mathrm{e} M \simeq$ $M^{\prime}$ e as $\mathbf{Z}_{\mathbf{c}}$-modules. Then,

(1) The space $\operatorname{Tor}_{\mathrm{H}_{\mathrm{c}}}^{\cdot}\left(M^{\prime}, N\right)$ is a graded commutative algebra and $\operatorname{Ext}_{\mathrm{H}_{\mathrm{c}}}(M, N)$ is a module over $\operatorname{Tor}_{\mathbf{H}_{\mathbf{c}}}\left(M^{\prime}, N\right)$. 
(2) The virtual de Rham differential on $\operatorname{Tor}_{\mathbf{H}_{\mathbf{c}}}\left(M^{\prime}, N\right)$ makes it into a $B V$ (=Batalin-Vilkovisky)-algebra so that $\operatorname{Ext}_{\mathrm{H}_{\mathrm{c}}}^{{ }_{\mathrm{c}}}(M, N)$ becomes a $B V$-module over the algebra $\operatorname{Tor}_{\mathbf{H}_{\mathbf{c}}}\left(M^{\prime}, N\right)$, again via the virtual de Rham differential.

The proof of Proposition 1 is explained in Section 5. We refer the reader to the appendix for the definition of BV-algebra.

\section{Verma modules}

Let $p \in \mathfrak{h}^{*}, W_{p}$ the stabilizer of $p$ under $W$ and $\lambda \in \operatorname{Irr}\left(W_{p}\right)$. Then $\lambda$ is a $\mathbb{C}\left[\mathfrak{h}^{*}\right] \rtimes W_{p^{-}}$ module, where $\mathbb{C}\left[\mathfrak{h}^{*}\right]$ acts by evaluation at $p$. Since $\mathbb{C}\left[\mathfrak{h}^{*}\right] \rtimes W_{p}$ is a subalgebra of $\mathbf{H}_{\mathbf{c}}$, we can define the induced module $\Delta(p, \lambda)=\mathrm{H}_{\mathbf{c}} \otimes_{\mathbb{C}\left[\mathfrak{h}^{*}\right] \rtimes W_{p}} \lambda$. Since the definition of $\Delta(p, \lambda)$ makes sense over $\mathrm{H}_{\mathbf{t}, \mathbf{c}}$, it is a quantizable module. It has recently been shown by Bonnafé and Rouquier [12] that each block $\Omega$ of the Calogero-Moser partition of $\operatorname{Irr}(W)$ has a canonical representative $\lambda_{\Omega}$. Combinatorially, it is defined in terms of the $b$-invariant; see Section 2. Now we can state the main result of this paper.

Theorem 2. Let $p \in \mathfrak{h}^{*}$ and $\Omega$ a CM-partition for $\left(W_{p}, \mathbf{c}^{\prime}\right)$.

(1) The quantizable module $\Delta\left(p, \lambda_{\Omega}\right)$ is simple.

(2) The canonical map $\mathbf{Z}_{\mathbf{c}} \rightarrow$ End $_{\mathrm{H}_{\mathbf{c}}}\left(\Delta\left(p, \lambda_{\Omega}\right)\right)$ is surjective.

(3) $\operatorname{Supp} \Delta\left(p, \lambda_{\Omega}\right) \subset X_{\mathbf{c}}^{\mathrm{sm}}$ if and only if $\Omega=\left\{\lambda_{\Omega}\right\}$.

When the support of $\Delta\left(p, \lambda_{\Omega}\right)$ is contained in the smooth locus of $X_{\mathbf{c}}$, one can show more. Namely, $E_{\mathbf{a}, \Omega}:=$ End $_{\mathbf{H}_{\mathbf{c}}}\left(\Delta\left(p, \lambda_{\Omega}\right)\right)$ is a polynomial ring and $\Delta(p, \lambda)$ is a free $E_{\mathbf{a}, \Omega}$-module of rank $|W|$. Here a is the image of $p$ in $\mathfrak{h}^{*} / W$. In general the Verma modules $\Delta(p, \lambda)$ are not simple if $\lambda$ is not the canonical representative in its Calogero-Moser partition, and its endomorphism ring is non-commutative.

As a consequence of Theorem 2 and Proposition 1, we deduce

Corollary 3. Let $M$ be a simple, quantizable module and $\Omega=\left\{\lambda_{\Omega}\right\}$ a $C M$ partition for $\left(W_{p}, \mathbf{c}^{\prime}\right)$. Then $\operatorname{Tor}_{\bullet}^{\mathrm{H}_{\mathbf{c}}}\left(\left(p, \lambda_{\Omega}^{*}\right) \Delta, M\right)$ is a $B V$-algebra and the space $\operatorname{Ext}_{\mathbf{H}_{\mathbf{c}}}^{\bullet}\left(\Delta\left(a, \lambda_{\Omega}\right), M\right)$ is a $B V$-module over the algebra $\operatorname{Tor}_{\bullet}^{\mathrm{H}_{\mathrm{c}}}\left(\left(p, \lambda_{\Omega}^{*}\right) \Delta, M\right)$.

\section{Self-intersections}

In particular, one can consider the derived self-intersections of the modules $\Delta\left(p, \lambda_{\Omega}\right)$. We assume for the remainder of this section that $\Omega=\left\{\lambda_{\Omega}\right\}$. Then the endomorphism ring $E_{\mathbf{a}, \Omega}$ is a quotient $\mathbf{Z}_{\mathbf{c}} / I$ of $\mathbf{Z}_{\mathbf{c}}$. Moreover, it is the coordinate ring of a smooth Lagrangian $\Lambda_{\mathbf{a}, \Omega} \simeq \mathbb{A}^{n}$. This means that $\mathrm{N}_{\mathbf{a}, \Omega}^{\vee}:=I / I^{2}$ is a free $E_{\mathbf{a}, \Omega^{-}}$ module. It is the module of sections of the conormal bundle of $\Lambda_{\mathbf{a}, \Omega}$ in $X_{\mathbf{c}}$. Its dual $\mathrm{N}_{\mathbf{a}, \Omega}:=\left(I / I^{2}\right)^{\vee}$ is the module of sections of the normal bundle of $\Lambda_{\mathbf{a}, \Omega}$ in $X_{\mathbf{c}}$. The following is an application of the theory developed in [2].

\section{Corollary 4.}

(1) There is an isomorphism of Gerstenhaber algebras

$$
\operatorname{Tor}_{\bullet}^{\mathrm{H}_{\mathrm{c}}}\left(\left(p, \lambda_{\Omega}^{*}\right) \Delta, \Delta\left(p, \lambda_{\Omega}\right)\right) \simeq \wedge^{\bullet} \mathrm{N}_{\mathbf{a}, \Omega}^{\vee} .
$$

(2) There is an isomorphism of Gerstenhaber modules

$$
\operatorname{Ext}_{\mathrm{H}_{\mathrm{c}}}\left(\Delta\left(p, \lambda_{\Omega}\right), \Delta\left(p, \lambda_{\Omega}\right)\right) \simeq \wedge^{\bullet} \mathrm{N}_{\mathbf{a}, \Omega}
$$


over the Gerstenhaber algebras in (1).

We outline the proof of Corollaries 3 and 4 in Section 5. The appendix contains a summary of the main results of [2] that are required in the article. The reader can also find the definition of Gerstenhaber algebra there. Corollary 4 implies that the virtual de Rham cohomology $H_{\text {vir }}^{\bullet}\left(\Delta\left(p, \lambda_{\Omega}\right), \Delta\left(p, \lambda_{\Omega}\right)\right)$ equals the usual de Rham cohomology of the conormal bundle of $\Lambda_{\mathbf{a}, \Omega}$ in $X_{\mathbf{c}}$; this is just $\mathbb{C}$ in degree zero since the space is contractible. Similarly, the virtual de Rham homology $H_{\bullet^{\text {vir }}}\left(\left(p, \lambda_{\Omega}^{*}\right) \Delta, \Delta\left(p, \lambda_{\Omega}\right)\right)$ is, up to a degree shift, also equal to the de Rham cohomology of the conormal bundle. See Corollary 28 for details.

\section{Motivation}

This paper was motivated by the close relationship, via the limit $t \rightarrow 0$ of Suzuki's functor [33], between the rational Cherednik algebra associated to the symmetric group $\mathfrak{S}_{n}$ and modules for the affine Lie algebra $\widehat{\mathfrak{g l}}_{m, \kappa}$ at the critical level $\kappa=-m$. If $\lambda$ is a partition of $n$ with at most $m$ parts then it is easy to see that this functor sends the Weyl module $\mathbb{V}(\lambda)$ to the Verma module $\Delta(\lambda)$. The endomorphism ring End $\widehat{\mathfrak{g l}}(\mathbb{V}(\lambda))$ is commutative and can be identified with the ring of functions on a certain moduli space of $G L_{m}$-opers on the formal disc. Moreover, the centre of $\widehat{\mathfrak{g l}}_{m, \kappa}$ surjects on to End $\widehat{\mathfrak{g r}}(\mathbb{V}(\lambda))$; see [20, Thm. 9.6.1]. This is a perfect analogue of our Theorem 2. By the result of Frenkel and Teleman [21], this identification, at least when $\lambda=0$, extends to an identification of $\operatorname{Ext}^{\bullet}(\mathbb{V}(0), \mathbb{V}(0))$ with the space of differential forms on the moduli space; a result completely analogous to our Corollary 4. Their (much more sophisticated) arguments also rely crucially on the fact that the vacuum module $\mathbb{V}(0)$ can be quantized, i.e., it exists for all levels $\kappa$.

\section{Rational Cherednik algebras at $t=0$}

\subsection{Definitions and notation}

Let $(W, \mathfrak{h})$ be a complex reflection group, where $\mathfrak{h}$ is the reflection representation for $W$, and let $\mathcal{S}(W)$ be the set of all complex reflections in $W$. For each $s \in \mathcal{S}(W)$, choose vectors $\alpha_{s} \in \mathfrak{h}$ and $\alpha_{s}^{\vee} \in \mathfrak{h}^{*}$ that span the one-dimensional spaces $\left.\operatorname{Im}(s-1)\right|_{\mathfrak{h}}$ and $\left.\operatorname{Im}(s-1)\right|_{\mathfrak{h}^{*}}$, respectively. We normalize $\alpha_{s}$ and $\alpha_{s}^{\vee}$ so that $\alpha_{s}^{\vee}\left(\alpha_{s}\right)=2$. Let c $: \mathcal{S}(W) \rightarrow \mathbb{C}$ be a $W$-equivariant function. The rational Cherednik algebra at $t=0$, as introduced by Etingof and Ginzburg [19], and denoted $\mathrm{H}_{\mathbf{c}}$, is the quotient of the skew group algebra of the tensor algebra $T\left(\mathfrak{h} \oplus \mathfrak{h}^{*}\right) \rtimes W$ by the ideal generated by the relations $\left[x, x^{\prime}\right]=\left[y, y^{\prime}\right]=0$ and

$$
[y, x]=\sum_{s \in \mathcal{S}} \mathbf{c}(s) \frac{x\left(\alpha_{s}\right) \alpha_{s}^{\vee}(y)}{\alpha_{s}^{\vee}\left(\alpha_{s}\right)} s,
$$

for all $x, x^{\prime} \in \mathfrak{h}^{*}$ and $y, y^{\prime} \in \mathfrak{h}$. By the PBW property, there is an isomorphism of vector spaces $\mathrm{H}_{\mathbf{c}} \simeq \mathbb{C}[\mathfrak{h}] \otimes \mathbb{C} W \otimes \mathbb{C}\left[\mathfrak{h}^{*}\right]$.

The trivial idempotent in $\mathbb{C} W$ is denoted $e$. Whenever $A$ is an algebra containing $\mathbb{C} W, E$ will denote the functor $A$-mod $\rightarrow e A e$-mod given by multiplication by $e$.

\subsection{The generalized Calogero-Moser space}

The centre $Z_{\mathbf{c}}$ of $\mathrm{H}_{\mathbf{c}}$ is an affine domain over which $\mathrm{H}_{\mathbf{c}}$ is a finite module. We shall denote by $X_{\mathbf{c}}:=$ Spec $Z_{\mathbf{c}}$ the corresponding affine variety. The space $X_{\mathbf{c}}$ is 
called the generalized Calogero-Moser space associated to the complex reflection group $W$ at parameter c. By [19, Prop. 4.15] we have inclusions $\mathbb{C}[\mathfrak{h}]^{W} \hookrightarrow \mathbf{Z}_{\mathbf{c}}$ and $\mathbb{C}\left[\mathfrak{h}^{*}\right]^{W} \hookrightarrow \mathbf{Z}_{\mathbf{c}}$. These inclusions define surjective morphisms $\pi: X_{\mathbf{c}} \rightarrow \mathfrak{h}^{*} / W$ and $\varpi: X_{\mathbf{c}} \rightarrow \mathfrak{h} / W$, respectively. Both $\pi$ and $\varpi$ are flat of relative dimension $\operatorname{dim} \mathfrak{h}$. Write

$$
\Upsilon: X_{\mathbf{c}} \rightarrow \mathfrak{h}^{*} / W \times \mathfrak{h} / W
$$

for the product morphism $\Upsilon:=\pi \times \varpi$. It is a finite, and hence closed, surjective morphism. By putting $x \in \mathfrak{h}^{*}$ in degree one, $y \in \mathfrak{h}$ in degree -1 and each $w \in W$ in degree zero, it is clear from the relations $(1)$ that $\mathrm{H}_{\mathbf{c}}$ is a $\mathbb{Z}$-graded algebra. This implies that $\mathbf{Z}_{\mathbf{c}}$ is also $\mathbb{Z}$-graded. Thus, there exists a canonical $\mathbb{C}^{\times}$-action on $X_{\mathbf{c}}$. The map $\Upsilon$ is $\mathbb{C}^{\times}$-equivariant since $\mathbb{C}[\mathfrak{h}]^{W} \otimes \mathbb{C}\left[\mathfrak{h}^{*}\right]^{W}$ is a graded subalgebra of $\mathbf{Z}_{\mathbf{c}}$. Proposition 4.15 of [19] implies that

Lemma 5. Let $\pi^{-1}(\mathbf{a})$ denote the scheme-theoretic fiber of $\pi$ over $\mathbf{a} \in \mathfrak{h}^{*} / W$. Then,

(1) The algebra $\mathbf{Z}_{\mathbf{c}}$ is free of rank $|W|$ over $\mathbb{C}[\mathfrak{h}]^{W} \otimes \mathbb{C}\left[\mathfrak{h}^{*}\right]^{W}$, hence $X_{\mathbf{c}}$ is CohenMacaulay.

(2) The algebra $\mathbb{C}\left[\pi^{-1}(\mathbf{a})\right]$ is a free $\mathbb{C}[\mathfrak{h}]^{W}$-module of rank $|W|$.

The affine scheme $\pi^{-1}(\mathbf{a})$ is neither reduced nor irreducible. The generalized Calogero-Moser space $X_{\mathbf{c}}$ has a natural Poisson structure; see [19].

\section{3. $\mathrm{H}_{\mathrm{c}}$-modules and fixed points of the $\mathbb{C}^{\times}$-action}

In this section we define Verma modules and recall some basic facts about the representation theory of rational Cherednik algebras. Detailed references for these facts can be found, for instance, in [6]. The stabilizer of $p \in \mathfrak{h}$, resp. $q \in \mathfrak{h}^{*}$, under $W$ is denoted $W_{p}$, resp. $W_{q}$.

Definition 1. The Verma module associated to $p$ and $\lambda \in \operatorname{Irr}\left(W_{p}\right)$ is the induced module

$$
\Delta(p, \lambda):=\mathrm{H}_{\mathbf{c}} \otimes_{\mathbb{C}\left[\mathfrak{h}^{*}\right] \rtimes W_{p}} \lambda,
$$

where the action of $\mathbb{C}\left[\mathfrak{h}^{*}\right]$ on $\lambda$ is via evaluation at $p$.

We write $\Delta(\lambda)$ for $\Delta(0, \lambda)$. Let $\mathfrak{m}_{\mathbf{b}} \triangleleft \mathbb{C}[\mathfrak{h}]^{W}$ the maximal ideal corresponding to the closed point $\mathbf{b} \in \mathfrak{h} / W$. The baby Verma module associated to $p, \lambda$ and $\mathbf{b}$ is defined to be

$$
\Delta(p, \lambda, \mathbf{b}):=\Delta(p, \lambda) / \mathfrak{m}_{\mathbf{b}} \cdot \Delta(p, \lambda) .
$$

The module $\Delta(0, \lambda, \mathbf{0})$ is the baby Verma module studied in [25]. If $L$ is an irreducible $\mathrm{H}_{\mathbf{c}}$-module then $\operatorname{dim} L \leq|W|$, with equality if and only if the support of $L$, a closed point of $X_{\mathbf{c}}$, is contained in the smooth locus. As noted above, the map $\Upsilon$ is $\mathbb{C}^{\times}$-equivariant. Since the image of 0 in $\mathfrak{h}^{*} / W \times \mathfrak{h} / W$ is the unique $\mathbb{C}^{\times}$fixed point of that space, the finitely many closed points of $\Upsilon^{-1}(0)$ are precisely the $\mathbb{C}^{\times}$-fixed points in $X_{\mathbf{c}}$. The irreducible $\mathrm{H}_{\mathbf{c}}$-modules supported at these fixed points are naturally parameterized by the set $\operatorname{Irr}(W)$. Namely, for each $\lambda \in \operatorname{Irr}(W)$, the module $\Delta(0, \lambda, \mathbf{0})$ has an irreducible head $L(\lambda)$. The irreducible modules $L(\lambda)$ can be graded, $L(\lambda) \neq L(\mu)$ for $\lambda \nsucc \mu$, and they exhaust all irreducible, gradable 
$\mathrm{H}_{\mathbf{c}}$-modules. If we stipulate that $L(\lambda)_{0}=\lambda$ and $L(\lambda)_{i}=0$ for all $i<0$, then this fixes the grading on $L(\lambda)$.

Our conventions about graded modules will be that $M[n]_{i}=M_{i-n}$. The coinvariant ring $\mathbb{C}[\mathfrak{h}]^{\operatorname{co} W}=\mathbb{C}[\mathfrak{h}] /\left\langle\mathbb{C}[\mathfrak{h}]_{+}^{W}\right\rangle$ is a graded copy of the regular representation, considered as a $W$-module. The fake polynomial associated to $\lambda \in \operatorname{Irr}(W)$ is

$$
f_{\lambda}(t)=\sum_{i \in \mathbb{Z}}\left[\mathbb{C}[\mathfrak{h}]_{i}^{\operatorname{co} W}: \lambda\right] t^{i},
$$

a polynomials in $\mathbb{N}[t]$. Define $b_{\lambda}$ to be the degree of the lowest non-zero monomial. It is called the $b$-invariant of $\lambda$.

\section{Graded modules}

Define $\mathbf{H}_{\mathbf{c}}^{+}=\mathbf{H}_{\mathbf{c}} /\left\langle\mathbb{C}\left[\mathfrak{h}^{*}\right]_{+}^{W}\right\rangle$ and, for each $\mathbf{b} \in \mathfrak{h} / W$, let $\mathrm{H}_{\mathbf{c}}^{\mathbf{b}}$ denote the quotient of $\mathrm{H}_{\mathbf{c}}^{+}$by the ideal generated by the maximal ideal defining $\mathbf{b}$ in $\mathfrak{h} / W$. Lemma 5 implies that $\operatorname{dim} \mathrm{H}_{\mathbf{c}}^{\mathbf{b}}=|W|^{3}$. When $\mathbf{b}=\mathbf{0}$, the algebra $\mathrm{H}_{\mathbf{c}}^{\mathbf{0}}$ is the restricted rational Cherednik algebra and the modules $L(\lambda)$ defined previously are $\mathbf{H}_{\mathbf{c}}^{\mathbf{0}}$-modules. The blocks of this algebra define a partition, the Calogero-Moser partition of $\operatorname{Irr}(W)$; $\operatorname{Irr}(W)=\bigcup_{i=1}^{k} \Omega_{i}$, where $L(\lambda)$ and $L(\mu)$ belong to the same block of $\mathrm{H}_{\mathbf{c}}^{\mathbf{0}}$ if and only if there is some $\Omega_{i}$ containing both $\lambda$ and $\mu$. We denote the set of all $\Omega_{i}$ by $\mathrm{CM}_{\mathbf{c}}(W)$. There is a natural bijection between $\mathrm{CM}_{\mathbf{c}}(W)$ and the closed points of $\Upsilon^{-1}(0,0)$.

Lemma 6. Let $\mathrm{H}_{\mathbf{c}}^{\mathbf{b}}=\bigoplus_{i=1}^{\ell} B_{i}$ be the block decomposition of $\mathbf{H}_{\mathbf{c}}^{\mathbf{b}}$

(1) For each $i$, there exists a unique irreducible module $L_{i} \in B_{i}$-mod such that $e L_{i} \neq 0$.

(2) We have $\operatorname{dim} e L_{i}=1$.

(3) Let $P_{i}$ be the projective cover of $L_{i}$. Then, $\operatorname{End}_{\mathrm{H}_{\mathrm{c}}^{\mathbf{b}}}\left(\bigoplus_{i=1}^{\ell} P_{i}\right) \simeq e \mathrm{H}_{\mathbf{c}}^{\mathbf{b}} e$ and the functor $E: \mathrm{H}_{\mathbf{c}}^{\mathbf{b}}-\bmod \rightarrow e \mathrm{H}_{\mathbf{c}}^{\mathbf{b}} e$-mod equals $\operatorname{Hom}_{\mathbf{H}_{\mathbf{c}}^{\mathbf{b}}}\left(\bigoplus_{i=1}^{\ell} P_{i},-\right)$.

Proof. By Müller's Theorem [13], the blocks of $\mathbf{H}_{\mathbf{c}}^{\mathbf{b}}$ are in bijection with the closed points of $\Upsilon_{\mathbf{c}}(\mathbf{b}, 0)$. Therefore, by [7, Lemma 4.4.1], there exists at least one irreducible module $L \in B_{i}$-mod such that $\operatorname{dim} e L=1$. The functor $E: \mathrm{H}_{\mathbf{c}}^{\mathbf{b}}$-mod $\rightarrow$ $e \mathrm{H}_{\mathbf{c}}^{\mathbf{b}} e$-mod, $E(M)=e M$, is a quotient functor with left adjoint ${ }^{\perp} E$ given by ${ }^{\perp} E(N)=\mathrm{H}_{\mathbf{c}}^{\mathbf{b}} e \otimes_{e \mathrm{H}_{\mathbf{c}}^{\mathbf{b}} e} N$. In particular, the adjunction id $\rightarrow E \circ{ }^{\perp} E$ is an isomorphism. If $L^{\prime}$ is another irreducible $B_{i}$-module with $e L \simeq e L^{\prime}$ as $e \mathrm{H}_{\mathbf{c}}^{\mathbf{b}} e$-modules, then ${ }^{\perp} E(L) \simeq{ }^{\perp} E\left(L^{\prime}\right)$. This implies that both $L$ and $L^{\prime}$ occur as composition factors of ${ }^{\perp} E(L)$. But then

$$
1=\operatorname{dim} e\left({ }^{\perp} E(L)\right) \geq\left[{ }^{\perp} E(L): L\right]+\left[{ }^{\perp} E(L): L^{\prime}\right] .
$$

This implies that $L \simeq L^{\prime}$, confirming (1).

Since $E$ is a quotient functor, it sends irreducible modules to irreducible modules. Therefore, since $e \mathrm{H}_{\mathbf{c}}^{\mathbf{b}} e$ is a commutative $\operatorname{ring}$, $\operatorname{dim} e M$ is at most one-dimensional for any irreducible module $M$. This proves (2).

Since $E$ is exact, Watt's Theorem says that there exists a projective $\mathrm{H}_{\mathbf{c}}^{\mathbf{b}}$-module $P$ such that $E \simeq \operatorname{Hom}_{\mathrm{H}_{\mathbf{c}}^{\mathbf{b}}}(P,-)$. The fact that $E\left(L_{i}\right)=e L_{i}$ and $E(L)=0$ for all other irreducible $B_{i}$-modules implies that $P=\bigoplus_{i=1}^{\ell} P_{i}$, proving (3). 
It has been shown in [12, Thm. 9.6.1] that each $\Omega \in \mathrm{CM}_{\mathbf{c}}(W)$ contains a unique representation with minimal $b$-invariant. This distinguished element in $\Omega$ is denoted $\lambda_{\Omega}$. The following is Theorem 9.6.1 of loc. cit.

Theorem 7. Let $\Omega \in \mathrm{CM}_{\mathbf{c}}(W)$.

(1) $\lambda_{\Omega}$ is the unique element in $\Omega$ such that $\operatorname{eL}\left(\lambda_{\Omega}\right) \neq 0$.

(2) $\operatorname{dim} e L\left(\lambda_{\Omega}\right)=1$.

(3) Let $B_{\Omega}$ be the block of $\mathrm{H}_{\mathbf{c}}^{0}$ corresponding to $\Omega$. Then, End $\mathbf{H}_{\mathbf{c}}\left(P\left(\lambda_{\Omega}\right)\right)=$ $e B_{\Omega} e$.

(4) The centre of $\mathrm{H}_{\mathbf{c}}^{\mathbf{0}}$ surjects onto End $\mathbf{H}_{\mathbf{c}}^{\mathbf{c}}\left(\Delta\left(0, \lambda_{\Omega}, \mathbf{0}\right)\right)$.

Remark 1. The functor $E: \mathrm{H}_{\mathbf{c}}^{0}-\bmod \rightarrow e \mathrm{H}_{\mathbf{c}}^{0} e-\bmod$ is isomorphic to $\operatorname{Hom}_{\mathrm{H}_{\mathbf{c}}^{\mathbf{0}}}\left(\mathrm{P}_{\mathbf{c}},-\right)$, where $\mathrm{P}_{\mathbf{c}}=\bigoplus_{\Omega \in \mathrm{CM}_{\mathbf{c}}(W)} P\left(\lambda_{\Omega}\right)$. Thus, it is strongly reminiscent of Soergel's $\mathbb{V}$ functor. Also, by choosing a linear character of the group $W$ other than the trivial representation, the analogue of Theorem 7 still holds, but the distinguished element in each block will be different.

Let $\mathrm{H}_{\mathbf{c}}^{+}$-grmod denote the category of finitely generated, $\mathbb{Z}$-graded $\mathrm{H}_{\mathbf{c}}^{+}$-modules, and similarly for $\mathrm{H}_{\mathbf{c}}^{\mathbf{0}}$-grmod, where in both cases homomorphisms are homogeneous of degree zero. Let $J_{\mathbb{Z}}\left(\mathrm{H}_{\mathbf{c}}^{+}\right)$denote the graded Jacobson radical of $\mathrm{H}_{\mathbf{c}}^{+}$, i.e., the intersection of all graded, maximal left ideals. Since $\mathbb{C}[\mathfrak{h}]_{+}^{W}$ is contained in $J_{\mathbb{Z}}\left(\mathrm{H}_{\mathbf{c}}^{+}\right)$, the inclusion $\mathrm{H}_{\mathbf{c}}^{0}$-grmod $\hookrightarrow \mathrm{H}_{\mathbf{c}}^{+}$-grmod induces a bijection between isomorphism classes of irreducible graded modules. For each $\mathbf{b} \in \mathfrak{h} / W$, let $(-)^{\mathbf{b}}: \mathrm{H}_{\mathbf{c}}^{+}-\bmod \rightarrow$ $\mathrm{H}_{\mathbf{c}}^{\mathbf{b}}$-mod be the functor $M \mapsto M^{\mathbf{b}}:=\mathrm{H}_{\mathbf{c}}^{\mathbf{b}} \otimes_{\mathrm{H}_{\mathbf{c}}^{+}} M$. To relate representations of $\mathrm{H}_{\mathbf{c}}^{+}$to representations of $\mathrm{H}_{\mathbf{c}}^{0}$, let $\mathrm{H}_{\mathbf{c}}^{+}$-pmod, resp. $\mathrm{H}_{\mathbf{c}}^{+}$-pgr, denote the category of projective $\mathrm{H}_{\mathrm{c}}^{+}$-modules, resp. graded, projective $\mathrm{H}_{\mathrm{c}}^{+}$-modules. These are exact categories. Then we have the following result, which is presumably standard.

\section{Lemma 8.}

(1) The abelian category $\mathrm{H}_{\mathbf{c}}^{+}$-grmod is Krull-Schmit.

(2) The irreducible module $L(\lambda)$ admits a projective cover $P^{+}(\lambda)$ in $\mathrm{H}_{\mathbf{c}}^{+}$-grmod.

(3) The $P^{+}(\lambda)[m]$ for $m \in \mathbb{Z}$ are all indecomposable projectives in $\mathrm{H}_{\mathbf{c}}^{+}$-pgr.

(4) The functor $(-)^{\mathbf{b}}: \mathrm{H}_{\mathbf{c}}^{+}$-pmod $\rightarrow \mathrm{H}_{\mathbf{c}}^{\mathbf{b}}(W)$-pmod is exact.

(5) The functor $(-)^{\mathbf{0}}: \mathrm{H}_{\mathbf{c}}^{+}-\mathrm{pgr} \rightarrow \mathrm{H}_{\mathbf{c}}^{\mathbf{0}}-\mathrm{pgr}$ is exact, essential surjective and induces a bijection between the blocks of $\mathrm{H}_{\mathbf{c}}^{+}$and the blocks of $\mathrm{H}_{\mathbf{c}}^{\mathbf{0}}$.

Proof. Since we were unable to find a suitable reference, we sketch the proof. As for the usual proof, e.g., [9, Sect. 1.4], of the Krull-Schmit property for Artinian rings, it suffices to show that the analogue of Fitting's Lemma holds in this setting. That is, given an indecomposable module $M \in \mathrm{H}_{\mathrm{c}}^{+}$-grmod and $f \in \operatorname{End}_{\mathrm{H}_{\mathrm{c}}^{+} \text {-grmod }}(M)$, we need to show that $M=\operatorname{Ker}\left(f^{n}\right) \oplus \operatorname{Im}\left(f^{n}\right)$ for some $n \gg 0$. Reading the usual proof of Fitting's Lemma, e.g., Lemma 1.4.4 of loc. cit., we just need to show that there is some $n \gg 0$ such that $\operatorname{Ker}\left(f^{n+k}\right)=\operatorname{Ker}\left(f^{n}\right)$ and $\operatorname{Im}\left(f^{n+k}\right)=\operatorname{Im}\left(f^{n}\right)$ for all $k$. Let $A$ be the degree zero part of $\mathrm{H}_{\mathbf{c}}^{+}$, a finite dimensional algebra. Since $M$ is finitely generated, there exists $N \gg 0$ such that $M=\mathrm{H}_{\mathbf{c}}^{+} \cdot M_{<N}$, and each weight space $M_{i}$ is a finite $A$-module. Therefore there is some $n \gg 0$ such that $\operatorname{Im}\left(f_{<N}^{n+k}\right)=\operatorname{Im}\left(f_{<N}^{n}\right)$, where $f_{<N}$ is $f$ restricted to $M_{<N}$. This implies that $\operatorname{Im}\left(f^{n+k}\right)=\operatorname{Im}\left(f^{n}\right)$. To show that $\operatorname{Ker}\left(f^{n+k}\right)=\operatorname{Ker}\left(f^{n}\right)$ too, consider the short exact sequences $0 \rightarrow \operatorname{Ker}\left(f_{i}^{n}\right) \rightarrow M_{i} \rightarrow \operatorname{Im}\left(f_{i}^{n}\right) \rightarrow 0$ and $0 \rightarrow \operatorname{Ker}\left(f_{i}^{n+k}\right) \rightarrow$ 
$M_{i} \rightarrow \operatorname{Im}\left(f_{i}^{n+k}\right) \rightarrow 0$ of $A$-modules. We certainly have $\operatorname{Ker}\left(f_{i}^{n+k}\right) \subset \operatorname{Ker}\left(f_{i}^{n}\right)$ and, by assumption, $\operatorname{Im}\left(f_{i}^{n}\right)=\operatorname{Im}\left(f_{i}^{n+k}\right)$. Since all $A$-modules have finite length, this implies that $\operatorname{Ker}\left(f_{i}^{n+k}\right)=\operatorname{Ker}\left(f_{i}^{n}\right)$ and hence $\operatorname{Ker}\left(f^{n+k}\right)=\operatorname{Ker}\left(f^{n}\right)$. This proves part (1).

Let $J_{\mathbb{Z}}\left(\mathrm{H}_{\mathbf{c}}^{+}\right)$denote the graded Jacobson radical of $\mathrm{H}_{\mathbf{c}}^{+}$. The ideal generated by $\mathbb{C}\left[\mathfrak{h}^{*}\right]_{+}^{W}$ is contained in $J_{\mathbb{Z}}\left(\mathrm{H}_{\mathbf{c}}^{+}\right)$. Therefore, the short exact sequence $0 \rightarrow$ $\left\langle\mathbb{C}\left[\mathfrak{h}^{*}\right]_{+}^{W}\right\rangle \rightarrow \mathrm{H}_{\mathbf{c}}^{+} \rightarrow \mathrm{H}_{\mathbf{c}}^{0} \rightarrow 0$ restricts to $0 \rightarrow\left\langle\mathbb{C}\left[\mathfrak{h}^{*}\right]_{+}^{W}\right\rangle \cap\left(\mathrm{H}_{\mathbf{c}}^{+}\right)_{0} \rightarrow\left(\mathrm{H}_{\mathbf{c}}^{+}\right)_{0} \rightarrow$ $\left(\mathrm{H}_{\mathbf{c}}^{\mathbf{0}}\right)_{0} \rightarrow 0$. Moreover, $\left\langle\mathbb{C}\left[\mathfrak{h}^{*}\right]_{+}^{W}\right\rangle \cap\left(\mathrm{H}_{\mathbf{c}}^{+}\right)_{0}$ is contained in the (ungraded) Jacobson radical of $\left(\mathrm{H}_{\mathrm{c}}^{+}\right)_{0}$. Therefore every idempotent (automatically homogeneous of degree zero) in $\mathrm{H}_{\mathbf{c}}^{\mathbf{0}}$ lifts to an idempotent in $\mathrm{H}_{\mathbf{c}}^{+}$. Let $a \in \mathrm{H}_{\mathbf{c}}^{\mathbf{0}}$ be a primitive idempotent, and $n(\lambda) \in \mathbb{Z}$, such that $\left(\mathrm{H}_{\mathbf{c}}^{\mathbf{0}} a\right)[n(\lambda)]$ is the projective cover of $L(\lambda)$ in $\mathrm{H}_{\mathbf{c}}^{\mathbf{0}}$-grmod. We denote by the same letter, the lift of $a$ to $\mathrm{H}_{\mathrm{c}}^{+}$. Then the claim is that $\left(\mathrm{H}_{\mathbf{c}}^{+} a\right)[n(\lambda)]$ is the projective cover of $L(\lambda)$ in $\mathrm{H}_{\mathbf{c}}^{+}$-grmod. It is certainly a graded projective module mapping surjectively onto $L(\lambda)$. If it is not the projective cover, then it is decomposable. Each summand is free over $\mathbb{C}[\mathfrak{h}]^{W}$, hence defines, after applying $(-)^{\mathbf{0}}$, a non-zero direct summand of $\left(\mathrm{H}_{\mathbf{c}}^{\mathbf{0}} a\right)[n(\lambda)]$. But this contradicts the indecomposability of the latter module. Parts (2) and (3) follow.

Since each $P \in \mathrm{H}_{\mathbf{c}}^{+}$-pmod is a direct summand of a direct sum of copies of $\mathrm{H}_{\mathbf{c}}^{+}$, it is a free $\mathbb{C}[\mathfrak{h}]^{W}$-module. This implies that $(-)^{\mathbf{b}}$ is exact. The fact that it maps projectives to projectives is just the adjunction $\operatorname{Hom}_{\mathbf{H}_{\mathbf{c}}^{\mathbf{b}}}\left(P^{\mathbf{b}}, M\right)=\operatorname{Hom}_{\mathrm{H}_{\mathbf{c}}^{+}}(P, M)$. This proves part (4).

Parts (3) and (4) imply the first part of (5). For the final statement about blocks, we note by part (3) that it suffices to show that $\operatorname{Hom}_{\mathrm{H}_{\mathrm{c}}^{+}}\left(P^{+}(\lambda), P^{+}(\mu)\right) \neq 0$ if and only if $\operatorname{Hom}_{\mathbf{H}_{\mathbf{c}}^{\mathbf{o}}}(P(\lambda), P(\mu)) \neq 0$. Since each $P^{+}(\lambda)$ is a direct summand of $\mathrm{H}_{\mathbf{c}}^{+}$, it is a graded free $\mathbb{C}[\mathfrak{h}]^{W}$-module. After fixing a grading on $P^{+}(\lambda)$ and $P^{+}(\mu)$, this makes $\operatorname{Hom}_{\mathrm{H}_{\mathrm{c}}^{+}}\left(P^{+}(\lambda), P^{+}(\mu)\right)$ into a graded $\mathbb{C}[\mathfrak{h}]^{W}$-module. Let $\mathbb{C}_{0}=$ $\mathbb{C}[\mathfrak{h}]^{W} / \mathbb{C}[\mathfrak{h}]_{+}^{W}$. Then, the sequence

$$
0 \rightarrow \mathbb{C}[\mathfrak{h}]_{+}^{W} \cdot P^{+}(\mu) \rightarrow P^{+}(\mu) \rightarrow \mathbb{C}_{0} \otimes_{\mathbb{C}[\mathfrak{h}]^{W}} P^{+}(\mu)=P(\mu) \rightarrow 0
$$

is exact, and applying $\operatorname{Hom}_{\mathrm{H}_{\mathrm{c}}^{+}}\left(P^{+}(\lambda),-\right)$, we deduce that $\operatorname{Hom}_{\mathrm{H}_{\mathbf{c}}^{\mathrm{o}}}(P(\lambda), P(\mu))$ equals $\mathbb{C}_{0} \otimes_{\mathbb{C}[\mathfrak{h}]^{W}} \operatorname{Hom}_{\mathrm{H}_{\mathrm{c}}^{+}}\left(P^{+}(\lambda), P^{+}(\mu)\right)$. By the graded Nakyama lemma, this implies that the space $\operatorname{Hom}_{\mathrm{H}_{\mathbf{c}}^{+}}\left(P^{+}(\lambda), P^{+}(\mu)\right)$ is non-zero if and only if the space $\operatorname{Hom}_{\mathbf{H}_{\mathbf{c}}^{\mathbf{c}}}(P(\lambda), P(\mu))$ is non-zero.

\subsection{Lifting}

We use Lemma 8 to lift the statements of Lemma 7 to $\mathrm{H}_{\mathbf{c}}^{+}$-mod.

Proposition 9. We have an isomorphism of exact functors

$$
E \simeq \bigoplus_{\Omega \in \mathrm{CM}_{\mathbf{c}}(W)} \operatorname{Hom}_{\mathrm{H}_{\mathrm{c}}^{+}}\left(P^{+}\left(\lambda_{\Omega}\right),-\right): \mathrm{H}_{\mathbf{c}}^{+}-\bmod \rightarrow e \mathrm{H}_{\mathbf{c}}^{+} e-\bmod
$$

Proof. The forgetful functor $\mathrm{H}_{\mathbf{c}}^{+}$-pgr $\rightarrow \mathrm{H}_{\mathbf{c}}^{+}$-pmod is essentially surjective. Therefore part (3) of Lemma 8 implies that there exist integers $n_{\lambda}$ such that $E \simeq$ $\bigoplus_{\lambda \in \operatorname{Irr}(W)} \operatorname{Hom}_{\mathrm{H}_{\mathrm{c}}^{+}}\left(P^{+}(\lambda)^{n_{\lambda}},-\right)$. Then $n_{\lambda}=\operatorname{dim} E(L(\lambda))$. But, by Theorem 7, $E(L(\lambda))=0$ if $\lambda$ is not the distinguished representative in its block and $\operatorname{dim} E\left(L\left(\lambda_{\Omega}\right)\right)=1$. 


\section{Endomorphism algebras}

Recall that the degrees of a complex reflection group $(W, \mathfrak{h})$ are the multiset of degrees of some (any) choice of homogeneous, algebraically independent generators of $\mathbb{C}[\mathfrak{h}]^{W}$. For brevity, we denote by $b_{\Omega}$, the $b$-invariant of $\lambda_{\Omega}^{*}$.

Theorem 10. Let $\Omega$ be a CM-partition and $\lambda_{\Omega}$ the distinguished representative. Then,

(1) The endomorphism ring $E_{\Omega}$ of the Verma module $\Delta\left(\lambda_{\Omega}\right)$ is an $\mathbb{N}$-graded quotient of $\mathrm{Z}_{\mathbf{c}}$. In particular, it is commutative.

(2) $e \Delta(\lambda)$ is a cyclic $E_{\Omega}$-module.

(3) The graded character of $E_{\Omega}$ is

$$
\operatorname{ch}_{q}\left(E_{\Omega}\right)=q^{-b_{\Omega}} f_{b_{\Omega}}(q) \prod_{i=1}^{n} \frac{1}{1-q^{d_{i}}}
$$

where $d_{1}, \ldots, d_{n}$ are the degrees of $(W, \mathfrak{h})$.

Proof. All the claims of the theorem follow more or less directly from Proposition 9. Let $B$ be the block of $\mathrm{H}_{\mathbf{c}}^{+}$corresponding to $\Omega$ and $P^{+}\left(\lambda_{\Omega}\right)$ the projective cover of $L\left(\lambda_{\Omega}\right)$ in $\mathrm{H}_{\mathbf{c}}^{+}$-grmod. Applying $\operatorname{Hom}_{\mathrm{H}_{\mathrm{c}}^{+}}\left(P^{+}\left(\lambda_{\Omega}\right),-\right)$ to the exact sequence $P^{+}\left(\lambda_{\Omega}\right) \rightarrow \Delta\left(\lambda_{\Omega}\right) \rightarrow 0$ gives a surjection of $e B e$-modules, $e B e \rightarrow e \Delta\left(\lambda_{\Omega}\right) \rightarrow 0$. Thus, $e \Delta\left(\lambda_{\Omega}\right)$ is a cyclic $e B e$-module. Since the action of $Z_{\mathbf{c}}$ on $e \Delta\left(\lambda_{\Omega}\right)$ factors through $e B e$, it is also a cyclic $\mathbf{Z}_{\mathbf{c}}$-module.

Similarly, applying $\operatorname{Hom}_{\mathrm{H}^{+}}\left(-, \Delta\left(\lambda_{\Omega}\right)\right)$ to $P^{+}\left(\lambda_{\Omega}\right) \rightarrow \Delta\left(\lambda_{\Omega}\right) \rightarrow 0$ gives an exact sequence $0 \rightarrow E_{\lambda_{\Omega}} \rightarrow e \Delta\left(\lambda_{\Omega}\right)$. Let $Z_{\Omega}$ denote the image of $Z_{\mathbf{c}}$ in $E_{\Omega}$. Then we have inclusions $\mathrm{Z}_{\Omega} \hookrightarrow E_{\Omega} \rightarrow e \Delta\left(\lambda_{\Omega}\right)$. These can be considered morphisms of finitely generated, graded $\mathbb{C}[\mathfrak{h}]^{W}$-modules. If $\mathbb{C}_{0}$ is the quotient of $\mathbb{C}[\mathfrak{h}]^{W}$ by its augmentation ideal, then Lemma 7 implies that morphism $\mathbb{C}_{0} \otimes_{\mathbb{C}[\mathfrak{h}]^{W}} Z_{\Omega} \rightarrow$ $\mathbb{C}_{0} \otimes_{\mathbb{C}[\mathfrak{h}]^{W}} e \Delta\left(\lambda_{\Omega}\right)$ is an isomorphism. Therefore, the graded Nakayama lemma implies that $\mathbf{Z}_{\Omega} \rightarrow e \Delta\left(\lambda_{\Omega}\right)$ is surjective. Thus, $\mathbf{Z}_{\Omega} \stackrel{\sim}{\longrightarrow} E_{\Omega} \stackrel{\sim}{\rightarrow} e \Delta\left(\lambda_{\Omega}\right)$. This implies parts (1) and (2).

Recall that $b_{\Omega}:=b_{\lambda_{\Omega}^{*}}$. As in the proof of [25, Thm. 5.6], an easy calculation shows that

$$
\operatorname{ch}_{q}\left(E_{\Omega}\right)=\operatorname{ch}_{q}\left(e \Delta(\lambda)\left[-b_{\Omega}\right]\right)=q^{-b_{\Omega}} f_{\Omega}(q) \prod_{i=1}^{n} \frac{1}{1-q^{d_{i}}} .
$$

Lemma 7 and Theorem 10 are false if $\lambda$ is not the distinguished representative in its block.

Remark 2. When $(\mathfrak{h}, W)=\left(\mathbb{C}^{n}, \mathfrak{S}_{n}\right)$ and $\mathbf{c} \neq 0$, we have

$$
\operatorname{ch}_{q}\left(E_{\lambda}\right)=\frac{1}{H_{\lambda}(q)}
$$

where $H_{\lambda}(q)$ is the hook polynomial of the partition $\lambda$.

When $\Omega=\left\{\lambda_{\Omega}\right\}$ consists of a single element, one can say a great deal more about the endomorphism ring $E_{\Omega}$. 
Lemma 11. If $\Omega=\left\{\lambda_{\Omega}\right\}$ then $\operatorname{Supp}(\Delta(\lambda))$ is contained in the smooth locus of $X_{\mathbf{c}}$.

Proof. As mentioned in Section 2, there is a natural bijection between the elements of $\mathrm{CM}_{\mathbf{c}}(W)$ and the points in $\Upsilon^{-1}(0,0)$. Let $\mathbf{x}_{\Omega}$ be the point corresponding to $\Omega$. The singular locus of $X_{\mathbf{c}}$ is $\mathbb{C}^{\times}$-stable. The module $\Delta(\lambda)$ is graded by putting $1 \otimes \lambda$ in degree zero. Then all non-zero weight spaces are positive. Therefore, if $I$ is the annihilator of $\Delta(\lambda)$ in $Z_{\mathbf{c}}$, then the quotient $Z_{\mathbf{c}} / I$ is positively graded with degree zero part equal to $\mathbb{C}$. This implies that $\operatorname{Supp} \Delta(\lambda)$ is contained inside the attracting set $\left\{x \in X_{\mathbf{c}} \mid \lim _{t \rightarrow \infty} t \cdot x=\mathbf{x}_{\Omega}\right\}$. The singular locus $X_{\mathbf{c}}^{\text {sing }}$ of $X_{\mathbf{c}}$ is $\mathbb{C}^{\times}$-stable. Therefore, if $\operatorname{Supp} \Delta(\lambda) \cap X_{\mathbf{c}}^{\text {sing }} \neq \varnothing$, then $\mathbf{x}_{\Omega}$ belongs to this intersection.

Corollary 12. If $\Omega=\left\{\lambda_{\Omega}\right\}$, then the commutative ring $E_{\Omega}$ is a graded polynomial ring.

Proof. The algebra $E_{\Omega}$ is $\mathbb{N}$-graded and connected. Therefore, there is a unique graded, maximal ideal $\mathfrak{m}$ in $E_{\Omega}$. The algebra $E_{\Omega}$ is a finite module over $\mathbb{C}[\mathfrak{h}]^{W}$. Therefore, by [8, Cor. 1.4.5], the Krull dimension of $E_{\Omega}$ is $\operatorname{dim} \mathfrak{h}$. Let $T=$ $\left(E_{+} / E_{+}^{2}\right)^{*}$ be the tangent space at the unique closed $\mathbb{C}^{\times}$-fixed point of $\operatorname{Spec}\left(E_{\Omega}\right)$. If we can show that $\operatorname{dim} T=n$ then the corollary follows from standard results on graded rings, e.g., [31, Thm. 1, Appendix III].

Let us show $\operatorname{dim} T=n$. It must have dimension at least $n$. $\operatorname{Since} \operatorname{Spec}\left(E_{\Omega}\right)$ is a closed subvariety of $X_{\mathbf{c}}, T$ is a subspace of $T_{\mathbf{x}_{\Omega}} X_{\mathbf{c}}$. The fact that $\mathbf{x}_{\Omega}$ is a $\mathbb{C}^{\times}$-fixed point implies that the space $T_{\mathbf{x}_{\Omega}} X_{\mathbf{c}}$ is a $\mathbb{C}^{\times}$-module and $T$ is a submodule. We decompose $T_{\mathbf{x}_{\Omega}} X_{\mathbf{c}}=T_{-} \oplus T_{0} \oplus T_{+}$, where $T_{-}$consists of all weight spaces of strictly negative weights, etc. We have $T \subset T_{+}$. Since $\mathbf{x}_{\Omega}$ is an isolated fixed point, it follows from [11, Cor. 2.2] that $T_{0}=0$. By assumption, $\mathbf{x}_{\Omega}$ is contained in the smooth locus of $X_{\mathbf{c}}$. Therefore, $T_{\mathbf{x}_{\Omega}} X_{\mathbf{c}}$ is a symplectic vector space. The symplectic form on $T_{\mathbf{x}_{\Omega}} X_{\mathbf{c}}$ is $\mathbb{C}^{\times}$-invariant. This implies that $\operatorname{dim} T_{+}=\operatorname{dim} T_{-}=n$. Thus, $T \subset T_{+}$implies that $\operatorname{dim} T \leq n$ as required.

Corollary 13. If $\Omega=\left\{\lambda_{\Omega}\right\}$, then the $E_{\Omega}$-module $\Delta\left(\lambda_{\Omega}\right)$ is free of rank $|W|$.

Proof. Since we have shown in Corollary 12 that $E_{\Omega}$ is a polynomial ring, and $\Delta\left(\lambda_{\Omega}\right)$ is a finitely generated $E_{\Omega}$-module, it suffices to show that $\Delta\left(\lambda_{\Omega}\right)$ is projective. Let $\mathfrak{m} \subset E_{\Omega}$ be a maximal ideal and consider the quotient $L=\Delta\left(\lambda_{\Omega}\right) / \mathfrak{m}$. $\Delta\left(\lambda_{\Omega}\right)$. This space is non-zero since any endomorphism $\phi \in E_{\Omega}$ whose image in $E_{\Omega} / \mathfrak{m} \cdot E_{\Omega}$ is non-zero induces a non-zero endomorphism of $L$. On the other hand, since $e \Delta\left(\lambda_{\Omega}\right)$ is a cyclic $E_{\Omega}$-module, the map

$$
E_{\Omega} / \mathfrak{m} \cdot E_{\Omega} \rightarrow e \Delta\left(\lambda_{\Omega}\right) / \mathfrak{m} \cdot e \Delta\left(\lambda_{\Omega}\right)=e L
$$

is an isomorphism. In particular, $\operatorname{dim} e L=1$. By Lemma 11, the support of $\Delta\left(\lambda_{\Omega}\right)$, and hence of $L$ too, is contained in the smooth locus of $X_{\mathbf{c}}$. Therefore, $L$ is irreducible and has dimension $|W|$. Thus, every fiber of $\Delta\left(\lambda_{\Omega}\right)$ has dimension $|W|$ over $E_{\Omega}$.

We have shown in Theorem 10 that $\mathrm{Z}_{\mathbf{c}}$ surjects on to End $\mathrm{H}_{\mathbf{c}}(\Delta(\lambda))$. 
Proposition 14. Assume that $X_{\mathbf{c}}$ is smooth. Then, multiplication defines a graded isomorphism

$$
\mathrm{Z}_{\mathbf{c}} / \sqrt{\left\langle\mathbb{C}[\mathfrak{h}]_{+}^{W}\right\rangle} \stackrel{\sim}{\longrightarrow} \operatorname{End}_{\mathrm{H}_{\mathbf{c}}}\left(\bigoplus_{\lambda \in \operatorname{Irr}(W)} \Delta(\lambda)\right) .
$$

Proof. The supports of the modules $\Delta(\lambda)$ are also disjoint because they are precisely the attracting sets for the $\mathbb{C}^{\times}$-action c.f. Lemma 11. Therefore the algebra End $H_{\mathbf{c}}\left(\bigoplus_{\lambda \in \operatorname{Irr}(W)} \Delta(\lambda)\right)$ is isomorphic to $\bigoplus_{\lambda \in \operatorname{Irr}(W)}$ End $_{\mathrm{H}_{\mathbf{c}}}(\Delta(\lambda))$. Theorem 10 (1) together with Corollary 12 implies that the support of $\Delta(\lambda)$ is reduced. Thus, $\sqrt{\left\langle\mathbb{C}[\mathfrak{h}]_{+}^{W}\right\rangle} \cdot \Delta(\lambda)=0$ for all $\lambda \in \operatorname{Irr}(W)$. These facts imply the statement of the proposition.

Remark 3. Even when $X_{\mathbf{c}}$ is smooth, one can show that $\pi^{-1}(0)$ is not reduced. In fact, it will be reduced if and only if $W \simeq \mathbb{Z}_{m}$ and $\mathbf{c}$ is generic. To see this, notice that Theorem 14 implies that $\mathbf{Z}_{\mathbf{c}} / \sqrt{\left\langle\mathbb{C}[\mathfrak{h}]_{+}^{W}\right\rangle}$ is positively graded. However, if $W$ has irreducible representations of dimension greater than one then $\mathbf{Z}_{\mathbf{c}} /\left\langle\mathbb{C}\left[\mathfrak{h}^{*}\right]_{+}^{W}\right\rangle$ has non-zero graded pieces of negative degree.

\section{Generalized Verma modules}

In this section we extend Theorem 10 to Verma modules for which $p \neq 0$. This is done by showing that the endomorphism ring of $\Delta(p, \lambda)$ is isomorphic to the endomorphism ring of the $\mathrm{H}_{\mathbf{c}^{\prime}}\left(W_{p}\right)$-module $\Delta(\lambda)$, where $\mathbf{c}^{\prime}$ denotes the restriction of $\mathbf{c}$ to the reflections in $W_{p}$ and $\mathrm{H}_{\mathbf{c}^{\prime}}\left(W_{p}\right)$ denotes the rational Cherednik algebra associated to $\left(\mathfrak{h}, W_{p}, \mathbf{c}^{\prime}\right)$. First, we recall a certain completion of $\mathbf{H}_{\mathbf{c}}(W)$, as defined by Bezrukavnikov and Etingof [10]. Our presentation will be slightly different from loc. cit., since it will be useful for the applications in [5] that it agrees with Wilson's factorization of the Calogero-Moser space when $W$ is the symmetric group.

Let $p \in \mathfrak{h}^{*}$ and let $\mathbf{a}$ be the image of $p$ in $\mathfrak{h}^{*} / W$. We denote by $\mathfrak{m}_{\mathbf{a}}$ the maximal ideal of $\mathbb{C}\left[\mathfrak{h}^{*}\right]^{W}$ corresponding to a. The completion of $\mathrm{H}_{\mathbf{c}}(W)$ with respect to the ideal generated by $\mathfrak{m}_{\mathbf{a}}$ is denoted $\widehat{\mathrm{H}}_{\mathbf{c}}(W)_{\mathbf{a}}$. The algebra $\mathbb{C}\left[\mathfrak{h}^{*}\right] /\left\langle\mathfrak{m}_{\mathbf{a}}\right\rangle$ is finite dimensional, with closed points corresponding to the $W$-orbit of $p$. Assume this orbit consists of $\ell$ points $p_{1}=p, p_{2}, \ldots, p_{\ell}$. Then, there exist primitive idempotents $\tilde{e}_{1}, \ldots, \tilde{e}_{\ell} \in \mathbb{C}\left[\mathfrak{h}^{*}\right] /\left\langle\mathfrak{m}_{\mathbf{a}}\right\rangle$ such that $\mathbb{C}\left[\mathfrak{h}^{*}\right] /\left\langle\mathfrak{m}_{\mathbf{a}}\right\rangle=\bigoplus_{i=1}^{\ell}\left(\mathbb{C}\left[\mathfrak{h}^{*}\right] /\left\langle\mathfrak{m}_{\mathbf{a}}\right\rangle\right) \cdot \tilde{e}_{i}$. Hensel's Lemma, [18, Cor. 7.5], implies that the primitive idempotents in

$$
\widehat{\mathbb{C}\left[\mathfrak{h}^{*}\right]_{\mathbf{a}}}:=\lim _{\leftarrow k} \mathbb{C}\left[\mathfrak{h}^{*}\right] /\left\langle\mathfrak{m}_{\mathbf{a}}^{k}\right\rangle
$$

are precisely the lifts $e_{i}$ of the $\tilde{e}_{i}$. Therefore,

$$
\widehat{\mathbb{C}\left[\mathfrak{h}^{*}\right]_{\mathbf{a}}}=\bigoplus_{i=1}^{\ell} \widehat{\mathbb{C}\left[\mathfrak{h}^{*}\right]_{\mathbf{a}}} \cdot e_{i}=\bigoplus_{i=1}^{\ell} \widehat{\mathbb{C}\left[\mathfrak{h}^{*}\right]_{p_{i}}}
$$

with each $\widehat{\mathbb{C}\left[\mathfrak{h}^{*}\right]_{\mathbf{p}}} \cdot e_{i}$ a local ring. For all $k \geq 0$, the PBW property for rational Cherednik algebras implies that

$$
\mathbb{C}\left[\mathfrak{h}^{*}\right] \cap\left(\mathfrak{m}_{\mathbf{a}}^{k} \cdot \mathrm{H}_{\mathbf{c}}(W)\right)=\mathfrak{m}_{\mathbf{a}}^{k} \cdot \mathbb{C}\left[\mathfrak{h}^{*}\right] .
$$


Hence, we have embeddings

$$
\mathbb{C}\left[\mathfrak{h}^{*}\right] /\left\langle\mathfrak{m}_{\mathbf{a}}^{k}\right\rangle \hookrightarrow \mathrm{H}_{\mathbf{c}}(W) / \mathfrak{m}_{\mathbf{a}}^{k} \cdot \mathrm{H}_{\mathbf{c}}(W)
$$

and taking the inductive limit, $\widehat{\mathbb{C}\left[\mathfrak{h}^{*}\right]_{\mathbf{a}}} \hookrightarrow \widehat{\mathrm{H}}_{\mathbf{c}}(W)_{\mathbf{a}}$, where we have used the fact that the functor of inverse limit is left exact. Therefore, we have $e_{i} \in \widehat{\mathrm{H}}_{\mathbf{c}}(W)_{\mathbf{a}}$ for all $i$ and

$$
\widehat{\mathrm{H}}_{\mathbf{c}}(W)_{\mathbf{a}}=\bigoplus_{i, j=1}^{\ell} e_{i} \widehat{\mathrm{H}}_{\mathbf{c}}(W)_{\mathbf{a}} e_{j}
$$

Let $\widehat{\mathrm{H}}_{\mathbf{c}^{\prime}}\left(W_{p_{i}}\right)_{p_{i}}$ denote the completion of $\mathbf{H}_{\mathbf{c}^{\prime}}\left(W_{p_{i}}\right)$ with respect to the maximal ideal $\mathfrak{n}_{p_{i}}$ in $\mathbb{C}\left[\mathfrak{h}^{*}\right]^{W_{p_{i}}}$. We write $\widetilde{\mathbb{C}\left[\mathfrak{h}^{*}\right]_{p_{i}}}$ for the completion of $\mathbb{C}\left[\mathfrak{h}^{*}\right]$ with respect to the maximal ideal $\mathfrak{n}_{p_{i}}$ in order to distinguish it from $\left.\widehat{\mathbb{C}\left[\mathfrak{h}^{*}\right]}\right]_{p_{i}}$. Write also $W_{i, j}$ for the subset of $W$ consisting of all elements $w$ such that $w \cdot e_{i}=e_{j}$. Before proving the main result of the section we require some preparatory lemmata.

Lemma 15. Multiplication defines a vector space isomorphism

$$
e_{i}\left(\mathbb{C} W_{i, j} \otimes \mathbb{C}[\mathfrak{h}]\right) \widehat{\otimes} \widetilde{\mathbb{C}\left[\mathfrak{h}^{*}\right]_{p_{j}}} e_{j} \stackrel{\sim}{\rightarrow} e_{i} \widehat{\mathrm{H}}_{\mathbf{c}}(W)_{b} e_{j}
$$

and hence

$$
e_{i} \widehat{\mathrm{H}}_{\mathbf{c}}(W)_{\mathbf{a}} e_{j} \otimes e_{j} \widehat{\mathrm{H}}_{\mathbf{c}}(W)_{\mathbf{a}} e_{k} \rightarrow e_{i} \widehat{\mathrm{H}}_{\mathbf{c}}(W)_{\mathbf{a}} e_{k}
$$

for all $1 \leq i, j, k \leq \ell$.

Proof. By standard results, e.g., [27, Sect. 7], we have canonical isomorphisms $\widehat{\mathbb{C}\left[\mathfrak{h}^{*}\right]_{\mathbf{a}}} \cdot e_{j} \simeq \widehat{\mathbb{C}\left[\mathfrak{h}^{*}\right]_{p_{j}}} \simeq \widetilde{\mathbb{C}\left[\mathfrak{h}^{*}\right]_{p_{j}}}$. Therefore, multiplication is a well-defined map. The algebra $\widehat{H}_{\mathbf{c}}(W)$ a is filtered by putting $\mathbb{C} W \otimes \widehat{\mathbb{C}\left[\mathfrak{h}^{*}\right]}$ a in degree zero and $\mathfrak{h}^{*} \subset \mathbb{C}[\mathfrak{h}]$ in degree one. The PBW property implies that the associated graded algebra is the skew group ring $(W \ltimes \mathbb{C}[\mathfrak{h}]) \widehat{\otimes} \widehat{\mathbb{C}\left[\mathfrak{h}^{*}\right]_{\mathbf{a}}}$ and the claim of the lemma on the level of associated graded spaces is clear. Since multiplication is a filtration preserving map it follows that it is also an isomorphism.

The second claim now follows from the fact that $\widetilde{\mathbb{C}\left[\mathfrak{h}^{*}\right]_{p_{j}}} e_{j} w=\widetilde{w \widetilde{\mathbb{C}\left[\mathfrak{h}^{*}\right]_{p_{k}}}} e_{k}$ for all $w \in W_{j, k}$ and the fact that $W_{i, j} \cdot W_{j, k}=W_{i, k}$.

Lemma 16. In $\widehat{\mathrm{H}}_{\mathbf{c}}(W)_{\mathbf{a}}$ we have $\left[x, e_{i}\right] e_{i}=0$, for all $x \in \mathfrak{h}^{*} \subset \mathbb{C}[\mathfrak{h}], 1 \leq i \leq \ell$.

Proof. Let $s \in W$ be a reflection. Recall the vectors $\alpha_{s} \in \mathfrak{h}$ and $\alpha_{s}^{\vee} \in \mathfrak{h}^{*}$ as defined in (1.1). It is shown in Section 3.5 of [25] that the functional $\alpha_{s}^{\vee}$ can be extended to a $\mathbb{C}$-linear operator on $\mathbb{C}\left[\mathfrak{h}^{*}\right]$ by setting

$$
\alpha_{s}^{\vee}\left(f f^{\prime}\right)=\alpha_{s}^{\vee}(f) f^{\prime}+f \alpha_{s}^{\vee}\left(f^{\prime}\right)-\lambda_{s} \frac{\alpha_{s}^{\vee}(f) \alpha_{s}^{\vee}\left(f^{\prime}\right)}{\alpha_{s}^{\vee}\left(\alpha_{s}\right)} \alpha_{s}, \quad \forall f, f^{\prime} \in \mathbb{C}\left[\mathfrak{h}^{*}\right] .
$$

This operator satisfies

$$
[x, f]=-\sum_{s \in S} \mathbf{c}(s) \frac{x\left(\alpha_{s}\right) \alpha_{s}^{\vee}(f)}{\alpha_{s}^{\vee}\left(\alpha_{s}\right)} s \quad \forall x \in \mathfrak{h}^{*} .
$$


It is also shown that $\alpha_{s}^{\vee}(f)=0$ for all $s \in \mathcal{S}$ and $f \in \mathbb{C}\left[\mathfrak{h}^{*}\right]^{W}$. Therefore $\alpha_{s}^{\vee}$ extends to an operator on $\widehat{\mathbb{C}\left[\mathfrak{h}^{*}\right]_{\mathbf{p}}}$ such that relation (4) holds for $f \in \widehat{\mathbb{C}\left[\mathfrak{h}^{*}\right]}$. Applying $\alpha_{s}^{\vee}$ to $e_{i}$ and using the fact that $e_{i}$ is an idempotent gives

$$
\alpha_{s}^{\vee}\left(e_{i}\right)\left(1-2 e_{i}+\lambda_{s} \frac{\alpha_{s}^{\vee}\left(e_{i}\right)}{\alpha_{s}^{\vee}\left(\alpha_{s}\right)} \alpha_{s}\right)=0 .
$$

Multiplying by $e_{i}$ and using the fact that $\widehat{\mathbb{C}\left[\mathfrak{h}^{*}\right]} e_{\mathbf{p}} \simeq \widehat{\mathbb{C}\left[\mathfrak{h}^{*}\right]_{p_{i}}}$, which is a domain, we must have

$$
e_{i} \alpha_{s}^{\vee}\left(e_{i}\right)=0 \quad \text { or } \quad e_{i} \alpha_{s}^{\vee}\left(e_{i}\right)=\frac{\lambda_{s}}{\alpha_{s}^{\vee}\left(\alpha_{s}\right)} \alpha_{s}^{-1} e_{i}
$$

However, $\alpha_{s}$ is invertible in the local ring $\left.\widehat{\mathbb{C}\left[\mathfrak{h}^{*}\right]}\right]_{p_{i}}$ if and only if $\alpha_{s}\left(p_{i}\right) \neq 0$ if and only if $s \cdot e_{i} \neq e_{i}$. Therefore, multiplying the expression for $\left[x, e_{i}\right]$ given in (4) on the right by $e_{i}$ gives zero.

Proposition 17. For $i=1, \ldots, \ell$ :

(1) we have an isomorphism of completed algebras

$$
\theta_{i}: \widehat{\mathrm{H}}_{\mathbf{c}^{\prime}}\left(W_{p_{i}}\right)_{p_{i}} \stackrel{\sim}{\longrightarrow} e_{i} \widehat{\mathrm{H}}_{\mathbf{c}}(W)_{\mathbf{a}} e_{i},
$$

(2) the functor $e_{i}: \widehat{\mathrm{H}}_{\mathbf{c}}(W)_{\mathbf{a}}-\bmod \rightarrow \widehat{\mathrm{H}}_{\mathbf{c}^{\prime}}\left(W_{p_{i}}\right)_{p_{i}}-\bmod , M \mapsto e_{i} M$ is an equivalence of categories,

(3) we have an isomorphism of commutative algebras

$$
\phi_{i}: Z\left(\widehat{\mathrm{H}}_{\mathbf{c}}(W)_{\mathbf{a}}\right) \stackrel{\sim}{\rightarrow} Z\left(\widehat{\mathrm{H}}_{\mathbf{c}^{\prime}}\left(W_{p_{i}}\right)_{p_{i}}\right) .
$$

Proof. By Lemma 15 we can define a map

$$
\theta_{i}: \widehat{\mathrm{H}}_{\mathbf{c}^{\prime}}\left(W_{p_{i}}\right)_{p_{i}}=\left(\mathbb{C} W_{i, i} \otimes \mathbb{C}[\mathfrak{h}]\right) \widehat{\otimes} \widetilde{\mathbb{C}\left[\mathfrak{h}^{*}\right]_{p_{i}}} \rightarrow e_{i} \widehat{\mathrm{H}}_{\mathbf{c}}(W)_{\mathbf{a}} e_{i},
$$

by $f \mapsto e_{i} f e_{i}$, which is an isomorphism of topological vector spaces. Note that $e_{i}$ commutes with elements from $\widetilde{\mathbb{C}\left[\mathfrak{h}^{*}\right]_{p_{i}}}$ and $W_{p_{i}}=W_{i, i}$. To show that this is an algebra morphism we must show that

$$
e_{i} x_{1} x_{2} e_{i}=e_{i} x_{1} e_{i} x_{2} e_{i}, \quad \forall x_{1}, x_{2} \in \mathfrak{h}^{*} \subset \mathbb{C}[\mathfrak{h}]
$$

and

$$
e_{i}[y, x]_{1} e_{i}=\left[e_{i} y e_{i}, e_{i} x e_{i}\right]_{2}, \quad \forall y \in \mathfrak{h}, x \in \mathfrak{h}^{*},
$$

where $[-,-]_{1}$ denotes the commutator in $\widehat{\mathrm{H}}_{\mathbf{c}^{\prime}}\left(W_{p_{i}}\right)_{p_{i}}$ and $[-,-]_{2}$ the commutator in $\widehat{\mathrm{H}}_{\mathbf{c}}(W)_{\mathbf{a}}$. The first equality follows from Lemma 16 and the second follows directly from the relations (1), noting that $e_{i}$ commutes with $y$.

The decomposition (3) of $\widehat{\mathrm{H}}_{\mathbf{c}}(W)_{\mathbf{a}}$ allows us to think of $\widehat{\mathrm{H}}_{\mathbf{c}}(W)_{\mathbf{a}}$ as a "matrix algebra". In particular, the centre of $\widehat{\mathrm{H}}_{\mathbf{c}}(W)_{\mathbf{a}}$ is contained in $\bigoplus_{i=1}^{\ell} e_{i} \widehat{\mathrm{H}}_{\mathbf{c}}(W)_{\mathbf{a}} e_{i}$ and the projection map $u_{i}: \widehat{\mathrm{H}}_{\mathbf{c}}(W)_{\mathbf{a}} \rightarrow e_{i} \widehat{\mathrm{H}}_{\mathbf{c}}(W)_{\mathbf{a}} e_{i}$ induces an isomorphism $u_{i}: Z\left(\widehat{\mathrm{H}}_{\mathbf{c}}(W)_{\mathbf{a}}\right) \rightarrow Z\left(e_{i} \widehat{\mathrm{H}}_{\mathbf{c}}(W)_{\mathbf{a}} e_{i}\right)$. Therefore, we have

$$
\phi_{i}=\theta_{i}^{-1} \circ u_{i}: Z\left(\widehat{\mathrm{H}}_{\mathbf{c}}(W)_{\mathbf{a}}\right) \stackrel{\sim}{\longrightarrow} Z\left(\widehat{\mathrm{H}}_{\mathbf{c}^{\prime}}\left(W_{p_{i}}\right)_{p_{i}}\right) .
$$


Corollary 18. Let $p \in \mathfrak{h}^{*}$ and $\mathbf{a} \in \mathfrak{h}^{*} / W$ as above. Let $\mathbf{a}_{i}$ be the image of $p_{i}$ in $\mathfrak{h}^{*} / W_{p_{i}}$. Then we have an isomorphism of schemes $\pi_{W}^{-1}(\mathbf{a}) \simeq \pi_{W_{p_{i}}}^{-1}\left(\mathbf{a}_{i}\right)$.

Proof. Since the isomorphism $\theta_{i}$ of Proposition 17 maps the space $\mathfrak{n}_{p_{i}}$ onto $e_{i} \mathfrak{m}_{\mathbf{a}} e_{i}$, the map $\phi_{i}$ of Proposition 17 satisfies

$$
\phi_{i}\left(\mathfrak{m}_{\mathbf{a}} \cdot Z\left(\widehat{\mathrm{H}}_{\mathbf{c}}(W)_{\mathbf{a}}\right)\right)=\mathfrak{n}_{p_{i}} \cdot Z\left(\widehat{\mathrm{H}}_{\mathbf{c}}\left(W_{p_{i}}\right)_{p_{i}}\right) .
$$

It is proved in [4, Lemma 3.9] that $Z\left(\widehat{\mathrm{H}}_{\mathbf{c}}(W)_{\mathbf{a}}\right)$ is the completion of $Z\left(\mathrm{H}_{\mathbf{c}}(W)\right)$ with respect to the ideal generated by $\mathfrak{m}_{\mathbf{a}}$ and, similarly, that $Z\left(\widehat{\mathrm{H}}_{\mathbf{c}^{\prime}}\left(W_{p_{i}}\right)_{p_{i}}\right)$ is the completion of $Z\left(\mathbf{H}_{\mathbf{c}^{\prime}}\left(W_{p_{i}}\right)\right)$ with respect to the ideal generated by $\mathfrak{n}_{p_{i}}$. Therefore, $\phi_{i}$ induces an isomorphism of commutative algebras

$$
\frac{Z\left(\mathrm{H}_{\mathbf{c}}(W)\right)}{\mathfrak{m}_{b} \cdot Z\left(\mathrm{H}_{\mathbf{c}}(W)\right)} \stackrel{Z}{\rightarrow} \frac{Z\left(\mathrm{H}_{\mathbf{c}^{\prime}}\left(W_{p_{i}}\right)\right)}{\mathbf{n}_{p_{i}} \cdot Z\left(\mathrm{H}_{\mathbf{c}^{\prime}}\left(W_{p_{i}}\right)\right)}
$$

The map $x \mapsto x, w \mapsto w$ and $y \mapsto y+y(p)$ for all $x \in \mathfrak{h}^{*} \subset \mathbb{C}[\mathfrak{h}], w \in W_{p}$ and $y \in \mathfrak{h} \subset \widehat{{\mathbb{C}\left[\mathfrak{h}^{*}\right.}_{p}}$ defines an isomorphism $\widehat{\mathrm{H}}_{\mathbf{c}^{\prime}}\left(W_{p}\right)_{p} \stackrel{\sim}{\rightarrow} \widehat{\mathrm{H}}_{\mathbf{c}^{\prime}}\left(W_{p}\right)_{0}$. Therefore, we will think of the functor $e_{1}: \widehat{\mathrm{H}}_{\mathbf{c}}(W)_{\mathbf{a}}$-mod $\rightarrow \widehat{\mathrm{H}}_{\mathbf{c}^{\prime}}\left(W_{p}\right)_{p}$-mod as an equivalence

$$
\Phi: \widehat{\mathrm{H}}_{\mathbf{c}}(W)_{\mathbf{a}}-\bmod \stackrel{\sim}{\longrightarrow} \widehat{\mathrm{H}}_{\mathbf{c}^{\prime}}\left(W_{p}\right)_{0} \text {-mod. }
$$

Now consider the generalized Verma module $\Delta(p, \lambda)$. Since $\mathfrak{m}_{\mathbf{a}} \cdot(1 \otimes \lambda)=0$ and $\mathfrak{m}_{\mathbf{a}}$ is central, $\Delta(p, \lambda)$ is a $\widehat{H}_{\mathbf{c}}(W)_{\mathbf{a}}$-module.

Lemma 19. Let $p \in \mathfrak{h}^{*}$ and $\lambda \in \operatorname{Irr}\left(W_{p}\right)$, then $\Phi(\Delta(p, \lambda)) \simeq \Delta(\lambda)$.

Proof. As a $\widehat{\mathrm{H}}_{\mathbf{c}}(W)_{\mathbf{a}}$-module,

$$
\Delta(p, \lambda)=\widehat{\mathrm{H}}_{\mathbf{c}}(W)_{\mathbf{a}} \otimes_{\widehat{\mathbb{C}\left[\mathfrak{h}^{*}\right]_{\mathbf{a}}} \rtimes W_{p}} \lambda=\bigoplus_{i, j=1}^{\ell} e_{i} \widehat{\boldsymbol{H}}_{\mathbf{c}}(W)_{\mathbf{a}} e_{j} \otimes_{\widehat{\mathbb{C}\left[\mathfrak{h}^{*}\right]_{\mathbf{a}}}} W_{p} \lambda .
$$

Recall that $\widehat{\mathbb{C}\left[\mathfrak{h}^{*}\right]_{\mathbf{a}}}$ acts on $\lambda$ by evaluation at $p$. Therefore, $e_{i} \cdot \lambda=0$ for all $i \neq 1$ and

$$
\widehat{\mathbb{C}\left[\mathfrak{h}^{*}\right]_{\mathbf{a}}} \rtimes W_{p}=\widehat{\mathbb{C}\left[\mathfrak{h}^{*}\right]_{\mathbf{a}}} e_{1} \rtimes W_{p} \oplus\left(\bigoplus_{i=2}^{\ell} \widehat{\mathbb{C}\left[\mathfrak{h}^{*}\right]_{\mathbf{a}}} e_{i}\right) \rtimes W_{p}
$$

implies that

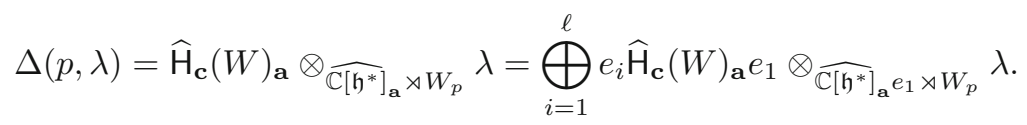

Thus,

$$
e_{1} \cdot \Delta(p, \lambda)=e_{1} \widehat{\mathrm{H}}_{\mathbf{c}}(W)_{\mathbf{a}} e_{1} \otimes_{\widehat{\mathbb{C}\left[\mathfrak{h}^{*}\right]_{\mathbf{a}}}} e_{1} \rtimes W_{p} \lambda .
$$

This implies that $\Phi(\Delta(p, \lambda)) \simeq \Delta(\lambda)$. 


\subsection{The proof of Theorem 2}

Since we have yet to define a simple quantizable module (this is done properly in Section 5), we simply note that in this case it means that $e \Delta\left(p, \lambda_{\Omega}\right)$ is a cyclic $\mathrm{Z}_{\mathbf{c}}$-module. We define $E_{\mathbf{a}, \Omega}:=\operatorname{End}_{\mathrm{H}_{\mathbf{c}}}\left(\Delta\left(p, \lambda_{\Omega}\right)\right)$. Since we can identify

$$
\operatorname{End}_{\mathrm{H}_{\mathbf{c}}}\left(\Delta\left(p, \lambda_{\Omega}\right)\right)=\operatorname{End}_{\widehat{\mathrm{H}}_{\mathbf{c}}}\left(\Delta\left(p, \lambda_{\Omega}\right)\right),
$$

Theorem 2 follows from Theorem 10, Proposition 17(2), and Lemma 19.

Again, if $\Omega=\left\{\lambda_{\Omega}\right\}$ in $\mathrm{CM}_{\mathbf{c}^{\prime}}\left(W_{p}\right)$, then the algebra $E_{\mathbf{a}, \Omega}$ is a polynomial ring of dimension $\operatorname{dim} \mathfrak{h}$. Let $w \in W$, then $\Delta(p, \lambda) \simeq \Delta(w(p), w(\lambda))$, where $w(\lambda)$ is the representation of $W_{w(p)}$ corresponding to $\lambda$ under the isomorphism $w: W_{p} \stackrel{\sim}{\longrightarrow}$ $W_{w(p)}$ of conjugation. Therefore, if a is the image of $p$ in $\mathfrak{h}^{*} / W$, then we denote by $\Lambda_{\mathbf{a}, \Omega}$ the support of the $\mathrm{Z}_{\mathbf{c}}$-module $\Delta(p, \lambda)$, thought of as a subscheme of $X_{\mathbf{c}}$. If $\Omega=\left\{\lambda_{\Omega}\right\}$, then $\Lambda_{\mathbf{a}, \Omega}$ is a smooth Lagrangian subvariety of $X_{\mathbf{c}}$, isomorphic to $\mathbb{A}^{n}$. The varieties $\Lambda_{\mathbf{a}, \Omega}$ play a key role in [5].

\subsection{An equivalence of categories}

Let $\mathbf{H}_{\mathbf{c}}-\bmod _{\mathbf{a}, \Omega}$ denote the category of finitely generated $\mathbf{H}_{\mathbf{c}}$-modules that are scheme-theoretically supported on $\Lambda_{\mathbf{a}, \Omega}$, i.e., those modules $M$ such that $I \cdot M=0$, where $I$ is the ideal defining $\Lambda_{\mathbf{a}, \Omega}$. The category of coherent $\mathcal{O}_{\Lambda_{\mathbf{a}, \Omega}}$-modules is denoted $\operatorname{Coh}\left(\Lambda_{\mathbf{a}, \Omega}\right)$. In this section we prove the following theorem:

Theorem 20. If $\Omega=\left\{\lambda_{\Omega}\right\}$, then the functor

$$
\left.F: \mathrm{H}_{\mathbf{c}}-\bmod _{\mathbf{a}, \Omega} \rightarrow \operatorname{Coh}\left(\Lambda_{\mathbf{a}, \Omega}\right), \quad F(M)=\operatorname{Hom}_{\mathbf{H}_{\mathbf{c}}} \widetilde{(\Delta(p, \lambda)}, M\right),
$$

is an equivalence of categories with quasi-inverse $N \mapsto G(N):=\Delta(p, \lambda) \otimes_{\mathrm{Z}_{\mathrm{c}}}$ $\Gamma\left(\Lambda_{\mathbf{a}, \Omega}, N\right)$.

Lemma 21. Let $e: \mathrm{H}_{\mathbf{c}}-\bmod \rightarrow \operatorname{Coh}\left(X_{\mathbf{c}}(W)\right)$ be the functor $M \mapsto \widetilde{e M}$.

(1) The functor $e$ is an equivalence if and only if $X_{\mathbf{c}}$ is smooth.

(2) If $\Omega=\left\{\lambda_{\Omega}\right\}$, then e defines an equivalence $\mathrm{H}_{\mathbf{c}}-\bmod _{\mathbf{a}, \Omega} \stackrel{\sim}{\longrightarrow} \operatorname{Coh}\left(\Lambda_{\mathbf{a}, \Omega}\right)$.

Proof. Part (1) is well-known, e.g., [19].

Part (2): by Lemma 11, the assumption of part (2) implies that $\Lambda_{\mathbf{a}, \Omega} \subseteq X_{\mathbf{c}}^{\mathrm{sm}}$. Since $\Lambda_{\mathbf{a}, \Omega} \cap X_{\mathbf{c}}^{\text {sing }}=\varnothing$, Hilbert's Nullstellensatz implies that $I\left(\Lambda_{\mathbf{a}, \Omega}\right)+I\left(X_{\mathbf{c}}^{\text {sing }}\right)=$ $\mathbf{Z}_{\mathbf{c}}$ and we can find a characteristic function $f \in \mathbf{Z}_{\mathbf{c}}$ taking the value 1 at all points of $\Lambda_{\mathbf{a}, \Omega}$ and vanishing on $X_{\mathbf{c}}^{\text {sing }}$. Replacing $\mathrm{H}_{\mathbf{c}}$ by its localization at $f$ and $\mathbf{Z}_{\mathbf{c}}$ by its localization, we may assume that $Z_{c}$ is a regular affine algebra and $H_{c}$ an Azumaya algebra over $\mathbf{Z}_{\mathbf{c}}$. We remark that part (1) still holds after localization. Recall that the centre $Z(\mathcal{A})$ of an abelian category $\mathcal{A}$ is defined to be the ring of endomorphisms End $\operatorname{A}_{\mathcal{A}}\left(\mathrm{id}_{\mathcal{A}}\right)$ of the identity functor. For a Noetherian $k$-algebra $A$, the centre of $A$-mod is canonically isomorphic to the centre of $A$. The equivalence $e$ induces an isomorphism $Z\left(\mathrm{H}_{\mathbf{c}}-\mathrm{mod}\right) \stackrel{\sim}{\longrightarrow} Z\left(\mathrm{Coh}\left(X_{\mathbf{c}}\right)\right)$ such that the composite

$$
Z\left(\mathrm{H}_{\mathbf{c}}\right) \stackrel{\sim}{\rightarrow} Z\left(\mathrm{H}_{\mathbf{c}}-\mathrm{mod}\right) \stackrel{\sim}{\longrightarrow} Z\left(\mathrm{Coh}\left(X_{\mathbf{c}}\right)\right) \stackrel{\sim}{\rightarrow} Z\left(\mathrm{H}_{\mathbf{c}}\right)
$$

is just the identity map. Let $I=I\left(\Lambda_{\mathbf{a}, \Omega}\right)$. We can identify $\mathrm{H}_{\mathbf{c}}-\bmod _{\mathbf{a}, \Omega}=\{M \in$ $\mathrm{H}_{\mathrm{c}}$-mod $\left.\mid i_{M}=0 \forall i \in I\right\}$, where $i_{M} \in$ End $_{\mathrm{H}_{\mathbf{c}}}(M)$ is the endomorphism defined by $i \in Z\left(\mathrm{H}_{\mathbf{c}}\right.$-mod). Similarly, $\operatorname{Coh}\left(\Lambda_{\mathbf{a}, \Omega}\right)=\left\{\mathcal{F} \in \operatorname{Coh}\left(X_{\mathbf{c}}\right) \mid i_{\mathcal{F}}=0 \forall i \in I\right\}$. From this it follows that $e: \mathrm{H}_{\mathbf{c}}-\bmod _{\mathbf{a}, \Omega} \stackrel{\sim}{\longrightarrow} \operatorname{Coh}\left(\Lambda_{\mathbf{a}, \Omega}\right)$.

Then, Theorem 20 follows from 
Lemma 22. The Verma module $\Delta\left(p, \lambda_{\Omega}\right)$ is a projective generator in $\mathrm{H}_{\mathbf{c}}-\bmod _{\mathbf{a}, \Omega}$.

Proof. By Lemma 21 it suffices to show that $e \widetilde{\Delta\left(p, \lambda_{\Omega}\right)}$ is a projective generator of $\operatorname{Coh}\left(\Lambda_{\mathbf{a}, \Omega}\right)$. But, by Theorem $2, e \Delta\left(p, \lambda_{\Omega}\right)$ is the regular representation as a $\mathbb{C}\left[\Lambda_{\mathbf{a}, \Omega}\right]$-module. Therefore $e \widehat{\Delta\left(p, \lambda_{\Omega}\right)} \simeq \mathcal{O}_{\Lambda_{\mathbf{a}, \Omega}}$ as sheaves on $\Lambda_{\mathbf{a}, \Omega}$.

Remark 4. If $I$ is the ideal of $\mathbf{Z}_{\mathbf{c}}$ defining $\Lambda_{\mathbf{a}, \Omega}$, then set $\mathrm{H}_{\mathbf{c}}(\mathbf{a}, \Omega):=\mathrm{H}_{\mathbf{c}} /\langle I\rangle$. One can reinterpret Lemma 22 as saying that $\mathrm{H}_{\mathbf{c}}(\mathbf{a}, \Omega)$ is a split Azumaya algebra over $\Lambda_{\mathbf{a}, \Omega}$, with splitting bundle $\Delta\left(p, \lambda_{\Omega}\right)$.

\subsection{Lagrangian subvarieties}

It is shown in [26, Prop. 4.5] that $X_{\mathbf{c}}$ is a symplectic variety; see [22] for the definition and properties of symplectic varieties. This implies that the smooth locus $X_{\mathbf{c}}^{\mathrm{sm}}$ is a symplectic leaf in $X_{\mathbf{c}}$ and hence its compliment has codimension at least two in $X_{\mathbf{c}}$.

Definition 2. A reduced subvariety $Y$ of $X_{\mathbf{c}}$ is said to a Lagrangian subvariety if $Y_{i}^{\mathrm{sm}} \cap X_{\mathbf{c}}^{\mathrm{sm}}$ is a non-empty Lagrangian submanifold of $X_{\mathbf{c}}^{\mathrm{sm}}$ for each irreducible component $Y_{i}$ of $Y$.

The goal of this subsection is to prove the following proposition. It is a consequence of Gabber's Integrability Theorem [23].

Proposition 23. Let $\mathbf{a} \in \mathfrak{h}^{*} / W$. Then $\pi^{-1}(\mathbf{a})_{\text {red }}$ is a Lagrangian subvariety of $X_{\mathbf{c}}$.

Let $\mathfrak{m}_{\mathbf{a}}$ be the maximal ideal in $\mathbb{C}\left[\mathfrak{h}^{*}\right]^{W}$ corresponding to a. First, we show

Lemma 24. Let $J$ be the radical of the ideal generated by $\mathfrak{m}_{\mathbf{a}}$ in $\mathbf{Z}_{\mathbf{c}}$. Then, $J$ is involutive, i.e., $\{J, J\} \subseteq J$.

Proof. It is clear from the definition of the Poisson bracket on $\mathbf{Z}_{\mathbf{c}}$ that the ideal generated by $\mathfrak{m}_{\mathbf{a}}$ in $\mathbf{Z}_{\mathbf{c}}$ is involutive. However, it seems that this does not in general imply that $J$ is involutive. Therefore, we need to work a bit harder. Since we have not assumed any smoothness condition on $X_{\mathbf{c}}$, we are also unable to use results from previous sections. Let $p_{1}, \ldots, p_{\ell} \in \mathfrak{h}^{*}$ be the elements in the orbit a. Set

$$
M=\bigoplus_{i=1}^{\ell}\left(\bigoplus_{\lambda \in W_{p_{i}}} \Delta\left(p_{i}, \lambda\right)\right) .
$$

Let $Y=(\operatorname{Supp} M)_{\text {red }}$. Then, I claim that $Y=V(J)$. Since $\mathfrak{m}_{\mathbf{a}} \cdot M=0$, we have $Y \subset V(J)$. Let $x \in V(J)$ and choose some irreducible $\mathrm{H}_{\mathbf{c}}$-module $L$ supported on $x$. As a $\mathbb{C}\left[\mathfrak{h}^{*}\right]$-module, $L=\oplus_{i=1}^{\ell} L_{p_{i}}$, where $L_{p_{i}}$ is supported at $p_{i}$. Without loss of generality, we may assume that $L_{p_{1}} \neq 0$. Let $\lambda \subset L_{p_{1}}$ be an irreducible $W_{p_{1}}{ }^{-}$ module in the socle of $L_{p_{1}}$. Then there is a non-zero homomorphism $\Delta\left(p_{1}, \lambda\right) \rightarrow L$. This implies that $\operatorname{Hom}_{\mathrm{H}_{\mathbf{c}}}(M, L) \neq 0$ and hence $x \in Y$.

Now, let $\mathbb{C}[\epsilon]$ be functions on the 3rd infinitesimal neighborhood of 0 in $\mathbb{C}$, so that $\epsilon^{3}=0$. It will be easier to work, via the Satake isomorphism, with the spherical subalgebra $e \mathrm{H}_{\mathbf{c}} e$. The usual rational Cherednik algebra $\mathrm{H}_{t, \mathbf{c}}$ has an additional parameter $t$, which we have assumed throughout is set to zero. Specializing instead to $t=\epsilon$, we have a $\mathbb{C}[\epsilon]$-algebra $e \mathrm{H}_{\epsilon, \mathbf{c}} e$ such that $e \mathrm{H}_{\epsilon, \mathbf{c}} e / \epsilon e \mathrm{H}_{\epsilon, \mathbf{c}} e \simeq e \mathrm{H}_{\mathbf{c}} e$. Then 
the Poisson structure on $e \mathrm{H}_{\mathbf{c}} e$ is constructed as in [23]. We can define $\Delta_{\epsilon}(p, \lambda)$ in the obvious way. It is a $\mathrm{H}_{\epsilon, \mathbf{c}}$-module, free over $\mathbb{C}[\epsilon]$. This gives us a $e \mathrm{H}_{\epsilon, \mathbf{c}} e$-module $e M_{\epsilon}$, free over $\mathbb{C}[\epsilon]$. This freeness implies that condition (1.2) of [23] is satisfied. Then, the fact that $J$ is involutive is a consequence of [23, Thm. II], together with the fact that the Satake isomorphism is an isomorphism of Poisson algebras.

Proof of Proposition 23. Let $n=\operatorname{dim} \mathfrak{h}$. The maximal ideal $\mathfrak{m}_{\mathbf{a}}$ in $\mathbb{C}[\mathfrak{h}]^{W}$ is generated by a regular sequence $f_{1}, \ldots, f_{n}$. By Lemma $5(1)$, they also form a regular sequence in $\mathbf{Z}_{\mathbf{c}}$. Therefore, the fact that the morphism $\pi$ is flat, together with [28, Cor. 9.6(ii)], implies that each irreducible component of $\pi^{-1}(\mathbf{a})_{\text {red }}$ is $n$-dimensional. Let $Y_{1}, \ldots, Y_{k}$ be these irreducible components. Let $\delta:=\prod_{s \in \mathcal{S}} \alpha_{s} \in \mathbb{C}[\mathfrak{h}]$; it is a semi-invariant with respect to $W$. Therefore, there exists some $k \in \mathbb{N}$ such that $\delta^{k} \in \mathbb{C}[\mathfrak{h}]^{W}$. The sequence $f_{1}, \ldots, f_{n}$ extends to a regular sequence $f_{1}, \ldots, f_{n}, \delta^{k}$ in $Z_{\mathbf{c}}$. This implies that, for each $i, \operatorname{dim} Y_{i} \cap V\left(\delta^{k}\right)=n-1$ if $Y_{i} \cap V\left(\delta^{k}\right) \neq \varnothing$. In particular, $Y_{i} \backslash V\left(\delta^{k}\right) \neq \varnothing$ for all $i$. The Dunkl embedding, as explained in [19, §4], shows that $\mathrm{H}_{\mathbf{c}}\left[\delta^{-k}\right] \simeq \mathbb{C}\left[\mathfrak{h} \times \mathfrak{h}_{\text {reg }}^{*}\right] \rtimes W$ where $\mathfrak{h}_{\text {reg }}^{*}$ is the set of points in $\mathfrak{h}^{*}$ with trivial $W$-stabilizer. Since the centre of $\mathbb{C}\left[\mathfrak{h} \times \mathfrak{h}_{\text {reg }}^{*}\right] \rtimes W$ is a regular domain, it follows that $Y^{i} \backslash V\left(\delta^{k}\right) \subset X_{\mathbf{c}}^{\mathrm{sm}}$. Thus, we have shown that $Y_{i} \cap X_{\mathbf{c}}^{\mathrm{sm}}$ is non-empty for all components $Y_{i}$ of $\pi^{-1}(\mathbf{a})_{\text {red }}$. Let $J_{i}$ be the ideal defining $Y_{i}$. It is a minimal prime over the ideal $J$. By Lemma $24, J$ is an involutive ideal. Therefore, $J_{i}$ is an involutive ideal; see [16, Lemma 2.1]. Choose some point $x \in Y_{i} \cap X_{\mathbf{c}}^{\mathrm{sm}}$. Then, $[15$, Prop. 1.5.1] says that $T_{x} Y_{i}$ is a coisotropic subspace of $T_{x} X_{\mathbf{c}}$. But $\operatorname{dim} T_{x} Y=n$, therefore it is actually a Lagrangian subspace as required.

\section{Remark 5.}

(1) If $X_{\mathbf{c}}$ is a smooth variety, then it is a symplectic manifold and Proposition 23 shows that $\pi^{-1}(\mathbf{a})_{\text {red }}$ is the disjoint union of finitely many Lagrangian submanifolds of $X_{\mathbf{c}}$.

(2) An analogous result to Proposition 23 holds when one considers $\varpi^{-1}$ (b) for $\mathbf{b} \in \mathfrak{h} / W$.

\section{Tor and Ext}

In this section we sketch the proof of the results stated in the introduction. A quantizable $\mathrm{H}_{\mathbf{c}}$-module $M$ is said to be simple if $e M$ is a cyclic $\mathbf{Z}_{\mathbf{c}}$-module.

Proof of Proposition 1. It will be easier to consider $\mathrm{H}_{\mathrm{c}}$ as a sheaf of algebras on $X_{\mathbf{c}}$. If $\widehat{\mathrm{H}}_{\mathbf{t}, \mathbf{c}}$ denotes the completion of $\mathrm{H}_{\mathbf{t}, \mathbf{c}}$ with respect to $(\mathbf{t})$, then via algebraic microlocalization [1], $\widehat{\mathrm{H}}_{\mathbf{t}, \mathbf{c}}$ is also a sheaf of algebras on $X_{\mathbf{c}}$. On the smooth locus $X_{\mathbf{c}}^{\mathrm{sm}}, E$ defines an equivalence $\mathrm{H}_{\mathbf{c}}-\bmod \stackrel{\sim}{\rightarrow} \mathbf{Z}_{\mathbf{c}}-\bmod$. This extends to an equivalence $\widehat{\mathrm{H}}_{\mathbf{t}, \mathbf{c}}-\bmod \stackrel{\sim}{\rightarrow} e \widehat{\mathrm{H}}_{\mathbf{t}, \mathbf{c}} e-\bmod$.

Since the support of $\left.M\right|_{X_{\mathbf{c}}^{\text {sm }}}$ equals the support of $\left.M^{\prime}\right|_{X_{\mathbf{c}}^{\text {sm }}}$, and we have assumed that $M$ and $N$ have smooth intersection,

$$
\operatorname{Tor}_{\bullet}^{\mathrm{H}}\left(M^{\prime}, N\right)=\operatorname{Tor}_{\bullet}^{\mathrm{H}}\left(\left.M^{\prime}\right|_{X_{\mathbf{c}}^{\mathrm{sm}}},\left.N\right|_{X_{\mathbf{c}}^{\mathrm{sm}}}\right) \simeq \operatorname{Tor}_{\bullet}^{\mathrm{Z}}\left(\left.e M\right|_{X_{\mathbf{c}}^{\mathrm{sm}}},\left.e N\right|_{X_{\mathbf{c}}^{\mathrm{sm}}}\right),
$$

and, similarly, $\operatorname{Ext}_{\mathbf{H}}^{\bullet}(M, N)$ is isomorphic to $\operatorname{Ext}_{Z}^{\bullet}\left(\left.e M\right|_{X_{\mathbf{c}}^{\text {sm }}},\left.e N\right|_{X_{\mathbf{c}}^{\text {sm }}}\right)$. Since $\left.e M\right|_{X_{\mathbf{c}}^{\text {sm }}}$ and $\left.e N\right|_{X_{\mathbf{c}}^{\text {sm }}}$ are simple, they are quotients of $\left.\mathbf{Z}_{\mathbf{c}}\right|_{X_{\mathbf{c}}^{\text {sm }}}$. Therefore, they are naturally 
commutative algebras. Now the claims of Proposition 1 are consequences of the theory developed in [2]; in particular, Corollary 1.1.3. The assumptions of loc. cit. that $\left.e M\right|_{X_{\mathbf{c}}^{\text {sm }}}$ and $\left.e N\right|_{X_{\mathbf{c}}^{\text {sm }}}$ define smooth, closed subvarities of $X_{\mathbf{c}}^{\text {sm }}$ is unnecessary in our case since that assumption is only required in order to guarantee the existence of a third order quantization of the modules. But, in our case, the existence of the quantization is built into the definition of quantizable modules.

Fix $p \in \mathfrak{h}^{*}, \Omega \in \mathrm{CM}_{\mathbf{c}^{\prime}}\left(W_{p}\right)$ and let a be the image of $p$ in $\mathfrak{h}^{*} / W$. Let $I$ be the ideal defining the closed subscheme $\Lambda_{\mathbf{a}, \Omega}$ in $X_{\mathbf{c}}$. We assume $\Omega=\left\{\lambda_{\Omega}\right\}$, so that $\Lambda_{\mathbf{a}, \Omega}$ is smooth. Then $\mathrm{N}_{\mathbf{a}, \Omega}^{\vee}:=I / I^{2}$ is a free $\mathbf{Z}_{\mathbf{c}} / I$-module. As noted in the introduction, it is the module of sections of the conormal bundle of $\Lambda_{\mathbf{a}, \Omega}$ in $X_{\mathbf{c}}$. Its dual $\mathrm{N}_{\mathbf{a}, \Omega}:=\left(I / I^{2}\right)^{\vee}$ is the module of sections of the normal bundle of $\Lambda_{\mathbf{a}, \Omega}$ in $X_{\mathrm{c}}$.

Proof of Corollary 4. Let $\lambda^{*}=\operatorname{Hom}_{\mathbb{C}}(\lambda, \mathbb{C})$, an irreducible right $W_{p}$-module. We denote by $\left(p, \lambda^{*}\right) \Delta$ the right $\mathrm{H}_{\mathbf{c}}$-module induced from the right $\mathbb{C}\left[\mathfrak{h}^{*}\right] \rtimes W_{p}$-module $\lambda^{*}$. Standard arguments using the Kozsul resolution show that $\operatorname{Ext}_{\mathbf{H}_{\mathbf{c}}}^{n}\left(\Delta(p, \lambda), \mathbf{H}_{\mathbf{c}}\right)$ $\simeq\left(p, \lambda^{*}\right) \Delta$, cf. [24, Lemma 4.1]. The results of Theorem 2 apply equally well to the right $\mathrm{H}_{\mathbf{c}}$-module $\left(p, \lambda^{*}\right) \Delta$. In order to be able to apply Proposition 1 , the only thing left to check is that $e \Delta(p, \lambda)$ and $\left(p, \lambda^{*}\right) \Delta e$ define the same smooth, closed subvariety of $X_{\mathbf{c}}$, i.e., that they are isomorphic as $\mathbf{Z}_{\mathbf{c}}$-modules. By completing $\mathrm{H}_{\mathbf{c}}$ at $\mathbf{b} \in \mathfrak{h}^{*} / W$, we may assume that $p=0$. Then, $\operatorname{Ext}_{\mathbf{H}_{\mathbf{c}}}^{n}\left(\Delta(\lambda), \mathrm{H}_{\mathbf{c}}\right) \simeq\left(\lambda^{*}\right) \Delta$ implies that the support of $\left(\lambda^{*}\right) \Delta$ is contained in the support of $\Delta(\lambda)$. In particular, it is contained in the smooth locus of $X_{\mathbf{c}}$. Thus, the "right block" of $\mathrm{H}_{\mathbf{c}}^{\mathbf{0}}$ containing $\lambda^{*}$ consists of a single element and Corollary 12 implies that the support of $\left(\lambda^{*}\right) \Delta$ is isomorphic to $\mathbb{A}^{n}$. Thus, $e \Delta(\lambda) \simeq\left(\lambda^{*}\right) \Delta e$ as $\mathrm{Z}_{\mathbf{c}}$-modules. The statement of the corollary now follows from Theorem 27 of the appendix.

Recall from Theorem 10 that the graded character of $\mathbb{C}\left[\Lambda_{\mathbf{0}, \Omega}\right]$ is equal to the product $q^{-b_{\Omega}} f_{\Omega}(q) \prod_{i=1}^{n}\left(1-q^{d_{i}}\right)^{-1}$, where $d_{1}, \ldots, d_{n}$ are the degrees of $(W, \mathfrak{h})$. Since $\mathbb{C}\left[\Lambda_{\mathbf{0}, \Omega}\right]$ is a polynomial ring, this implies that there exist ${ }^{1}$ integers $0<e_{1} \leq$ $\cdots \leq e_{n}$ such that

$$
q^{-b_{\Omega}} f_{\Omega}(q) \prod_{i=1}^{n} \frac{1}{1-q^{e_{i}}}=\prod_{i=1}^{n} \frac{1}{1-q^{d_{i}}} .
$$

Corollary 25. When $\Omega=\left\{\lambda_{\Omega}\right\}$, we have, as bigraded vector spaces,

$$
\operatorname{ch}_{q, t}\left(\operatorname{Tor}_{\bullet}^{\mathrm{H}_{\mathrm{c}}}((\lambda) \Delta, \Delta(\lambda))\right)=q^{-b_{\Omega}} f_{\Omega}(q) \prod_{i=1}^{n} \frac{1+t q^{-e_{i}}}{1-q^{d_{i}}}
$$

and

$$
\operatorname{ch}_{q, t}\left(\operatorname{Ext}_{\mathrm{H}_{\mathbf{c}}}^{\cdot}(\Delta(\lambda), \Delta(\lambda))\right)=q^{-b_{\Omega}} f_{\Omega}(q) \prod_{i=1}^{n} \frac{1+t q^{e_{i}}}{1-q^{d_{i}}} .
$$

\footnotetext{
${ }^{1}$ For an arbitrary representation $\lambda \in \operatorname{Irr}(W)$, it is not possible to find integers $0<$ $e_{1} \leq \cdots \leq e_{n}$ such that (5) holds. This is related to the fact that $\mathbf{x}_{\lambda}$ is always in the singular locus of $X_{\mathbf{c}}$, regardless of the parameter $\mathbf{c}$.
} 
Proof. Let $x$ be the $\mathbb{C}^{\times}$-fixed point in $X_{\mathbf{c}}$ over which $L\left(\lambda_{\Omega}\right)$ lives. As in the proof of Corollary 12, the integers $e_{i}$ are defined so that the graded character of $T_{x} \Lambda_{\mathbf{0}, \Omega}$ is given by $\sum_{i=1}^{n} q^{-e_{i}}$. We have $T_{x} X_{\mathbf{c}}=T_{x} \Lambda_{\mathbf{0}, \Omega} \oplus\left(T_{x} \Lambda_{\mathbf{0}, \Omega}\right)^{\perp}$ with respect to the symplectic form on $X_{\mathbf{c}}$, and we may identify $\left(T_{x} \Lambda_{\mathbf{0}, \Omega}\right)^{\perp}=\left(T_{\Lambda_{\mathbf{0}, \Omega}} X_{\mathbf{c}}\right)_{x}$ as $\mathbb{C}^{\times}$-representations. This implies that

$$
\operatorname{ch}_{q}\left(T_{\Lambda_{\mathbf{0}, \Omega}} X_{\mathbf{c}}\right)_{x}=\sum_{i=1}^{n} q^{e_{i}} .
$$

Then formula (7) follows from the fact that

$$
\operatorname{Ext}_{\mathrm{H}_{\mathbf{c}}}\left(\Delta\left(\lambda_{\Omega}\right), \Delta\left(\lambda_{\Omega}\right)\right)=\mathbb{C}\left[\Lambda_{\mathbf{0}, \Omega}\right] \otimes \wedge^{\bullet}\left(T_{\Lambda_{\mathbf{0}, \Omega}} X_{\mathbf{c}}\right)_{x}
$$

as graded vector spaces, together with the fact that if $V$ is a graded vector space with character $q^{m_{1}}+\cdots+q^{m_{k}}$ then the bigraded character of $\wedge^{\bullet} V$ is given by $\left(1+t q^{m_{1}}\right) \cdots\left(1+t q^{m_{k}}\right)$. The proof of formula (6) is similar.

We end the section by noting one other situation were one can easily calculate virtual deRham (co)homology groups. Let $q \in \mathfrak{h}$ and $\mu \in \operatorname{Irr}\left(W_{q}\right)$. We let the dual Verma module associated to $q$ and $\mu$ be the induced module $\nabla(q, \mu):=$ $\mathrm{H}_{\mathbf{c}} \otimes_{\mathbb{C}[\mathfrak{h}] \rtimes W_{q}} \mu$, where the action of $\mathbb{C}[\mathfrak{h}]$ on $\mu$ is via evaluation at $q$. Choose $\Omega \in \mathrm{CM}_{\mathbf{c}}(W)$ such that $\Omega=\left\{\lambda_{\Omega}\right\}$. Let $\mathbb{C}[i]$ denote the complex with a copy of $\mathbb{C}$ in degree $i$ and zero in all other degrees.

Proposition 26. We have

$$
\operatorname{Tor}_{\bullet}^{\mathrm{H}_{\mathrm{c}}}\left(\left(\lambda_{\Omega}\right) \Delta, \nabla\left(\lambda_{\Omega}\right)\right) \simeq \mathbb{C}[0] \text { and } \operatorname{Ext}_{\mathrm{H}_{\mathrm{c}}}\left(\Delta\left(\lambda_{\Omega}\right), \nabla\left(\lambda_{\Omega}\right)\right) \simeq \mathbb{C}[n] \text {. }
$$

Proof. Our assumptions on $\Omega$ imply that there is a regular sequence of homogeneous elements $\mathbf{z}=z_{1}, \ldots, z_{n}$ such that $\Lambda_{\mathbf{0}, \Omega}=V(\mathbf{z})$. Let $\Pi_{\Omega}$ denote the support of $\nabla\left(\lambda_{\Omega}\right)$. The analogue of Theorem 2 in this context implies that $\Pi_{\Omega}$ is a smooth Lagrangian subvariety of $X_{\mathbf{c}}$, isomorphic to $\mathbb{A}^{n}$. Also, $\operatorname{Tor}_{\bullet}^{\mathrm{H}}\left(\left(\lambda_{\Omega}\right) \Delta, \nabla\left(\lambda_{\Omega}\right)\right) \simeq$ $\operatorname{Tor}_{\bullet}^{X_{\mathbf{c}}}\left(\mathcal{O}_{\Lambda_{\mathbf{0}, \Omega}}, \mathcal{O}_{\Pi_{\Omega}}\right)$ and $\operatorname{Ext}_{\mathbf{H}}^{\bullet^{\prime}}\left(\Delta\left(\lambda_{\Omega}\right), \nabla\left(\lambda_{\Omega}\right)\right) \simeq \operatorname{Ext}_{X_{\mathbf{c}}}\left(\mathcal{O}_{\Lambda_{\mathbf{0}, \Omega}}, \mathcal{O}_{\Pi_{\Omega}}\right)$, so it suffices to calculate the latter groups. It is explained in Chapter 17 of [18] how one does this. We use the notation of loc. cit. Using the fact that both $\Lambda_{\mathbf{0}, \Omega}$ and $\Pi_{\Omega}$ are smooth subvarieties of $X_{\mathbf{c}}$ intersecting transversely in a single point, we have

$$
\operatorname{Tor}_{\bullet}^{X_{\mathbf{c}}}\left(\mathcal{O}_{\Lambda_{\mathbf{0}, \Omega}}, \mathcal{O}_{\Pi_{\Omega}}\right)=H_{\bullet}\left(\mathbf{z}, \mathcal{O}_{\Pi_{\Omega}}\right) \simeq \mathbb{C}[0],
$$

and $\operatorname{Ext}_{X_{\mathbf{c}}}\left(\mathcal{O}_{\Lambda_{\mathbf{0}, \Omega}}, \mathcal{O}_{\Pi_{\Omega}}\right)=H^{\bullet}\left(\mathbf{z}, \mathcal{O}_{\Pi_{\Omega}}\right)=H_{n-\bullet}\left(\mathbf{z}, \mathcal{O}_{\Pi_{\Omega}}\right) \simeq \mathbb{C}[n]$, as required.

Obviously, $H_{\text {vir }}^{\cdot}\left(\Delta\left(\lambda_{\Omega}\right), \nabla\left(\lambda_{\Omega}\right)\right)=\mathbb{C}[n]$ and $H_{\bullet}^{\operatorname{vir}}\left(\left(\lambda_{\Omega}^{*}\right) \Delta, \nabla\left(\lambda_{\Omega}\right)\right)=\mathbb{C}$ in this case.

\section{Remarks}

By Lemma 8 (5), the connected components of $\varpi^{-1}(\mathbf{0})_{\text {red }}$ are naturally labeled by the elements of $\mathrm{CM}_{\mathbf{c}}(W)$. Moreover, Lemma 5 implies that the irreducible components of $\varpi^{-1}(\mathbf{0})_{\text {red }}$ are dim $\mathfrak{h}$-dimensional. Based on examples, it seems natural to conjecture:

(1) Each connected component of $\varpi^{-1}(\mathbf{0})_{\text {red }}$ is irreducible and isomorphic to $\mathbb{A}^{n}$, where $n=\operatorname{dim} \mathfrak{h}$; i.e., $\varpi^{-1}(\mathbf{0})_{\text {red }}=\bigsqcup_{\Omega} \mathbb{A}_{\Omega}^{n}$. 
(2) For each $\Omega \in \mathrm{CM}_{\mathbf{c}}(W), E_{\Omega}=\mathbb{C}\left[\mathbb{A}_{\Omega}^{n}\right]$ as quotients of $\mathbf{Z}_{\mathbf{c}}$.

(3) The map $\pi \mid: \mathbb{A}_{\Omega}^{n} \rightarrow \mathfrak{h} / W$ is flat of degree $\operatorname{dim} \lambda_{\Omega}$, and is generically a covering.

We note that if $Y$ is the connected component of $\varpi^{-1}(\mathbf{0})_{\text {red }}$ labeled by $\Omega$, then one can study the generic fibers of $\pi \mid: Y \rightarrow \mathfrak{h} / W$ by considering the representation theory of $\mathbf{H}_{\mathbf{c}}^{\mathbf{b}}$. Namely, statements (2) and (3) above would imply the following:

(4) Assume that $\mathbf{b}$ is generic. Then the head of $P^{+}\left(\lambda_{\Omega}\right)^{\mathbf{b}}$ equals $\Delta\left(0, \lambda_{\Omega}, \mathbf{b}\right), \mathbf{a}$ semi-simple module, with pairwise non-isomorphic irreducible summands.

\section{Appendix: Batalin-Vilkoviski structures}

In this appendix we summarize the main results of [2], as required in this article. We follow the presentation of loc. cit., which the reader is encouraged to consult for further details. All undecorated tensor products will mean tensor products over $\mathbb{C}$.

Let $D=\bigoplus_{i>0} D_{i}$ be a graded commutative algebra. If $D$ is equipped with a differential $\delta: D_{\bullet} \rightarrow D_{\bullet-1}, \delta^{2}=0$, and

$$
\begin{aligned}
\delta(a b c)= & \delta(a b) c+(-1)^{\operatorname{deg}(a)} a \delta(b c)+(-1)^{(\operatorname{deg}(a)+1) \operatorname{deg}(b)} b \delta(a c)-\delta(a) b c \\
& -(-1)^{\operatorname{deg}(a)} a \delta(b) c-(-1)^{\operatorname{deg}(a)+\operatorname{deg}(b)} a b \delta(c)+\delta(1) a b c,
\end{aligned}
$$

for all homogeneous elements $a, b, c$ of $D$, then $D$ is said to be a Batalin-Vilkoviski (BV) algebra. The above equation is equivalent to the fact that $\delta$ is a differential operator of order at most two. Every Batalin-Vilkoviski algebra has in addition the structure of a Gerstenhaber algebra. Namely, for $x, y$ homogeneous elements in $D$, the formula

$$
[x, y]:=\delta(x y)-\delta(x) y-(-1)^{\operatorname{deg} x} x \delta(y)
$$

makes $D$. into a Gerstenhaber algebra. This means that $[-,-]$ has degree -1 and

$$
\begin{aligned}
{[a, b c] } & =[a, b] c+(-1)^{(\operatorname{deg}(a)-1) \operatorname{deg}(b)} b[a, c], \\
{[a, b] } & =-(1)^{(\operatorname{deg}(a)-1)(\operatorname{deg}(b)-1)}[b, a], \\
{[a,[b, c]] } & =[[a, b], c]+(-1)^{(\operatorname{deg}(a)-1)(\operatorname{deg}(b)-1)}[b,[a, c]],
\end{aligned}
$$

for all homogeneous elements $a, b, c$ of $D$, i.e., the graded commutative algebra $D$ is an odd Poisson algebra.

Let $X$ be a smooth affine variety equipped with a symplectic two-form $\omega$. Let $Y$ be a smooth, coisotropic subvariety of $X$. Let $\mathrm{N}_{X / Y}$ denote the sheaf of sections of the normal bundle of $Y$ in $X$. It is a sheaf of $\mathcal{O}_{X}$-modules supported on $Y$. Its dual $\mathrm{N}_{X / Y}^{\vee}$ is the sheaf of sections of the conormal bundle. Since the two-form $\omega$ is non-degenerate, it induces an isomorphism $\Gamma\left(X, \Omega_{X}^{2}\right) \simeq \Gamma\left(X, \wedge^{2} \Theta_{X}\right)$. We let $P$ be the image of $\omega$ under this isomorphism; it is a Poisson bivector. The graded commutative algebras $\wedge^{\bullet} N_{X / Y}^{\vee}$ and $\wedge^{\bullet} N_{X / Y}$ have a natural structure of BV algebra. Namely, the differential on $\wedge^{\bullet} \mathrm{N}_{X / Y}^{\vee}$ is given by the formula $\delta=$ $i_{P} \circ d_{\mathrm{DR}}+d_{\mathrm{DR}} \circ i_{P}$, where $d_{\mathrm{DR}}$ is the deRham differential of degree one and $i_{P}$ denotes contraction with $P$ (and thus $\delta$ has degree -1 ). The differential on $\wedge \bullet N_{X / Y}$ is given by the Schouten bracket $[P,-]$. That these operations are indeed well defined follows from the fact that the ideal defining $Y$ is involutive. Combining Corollary 1.1.3, Propositions 5.1.1 and 5.2.1 of [2] gives 
Theorem 27. The sheaf of graded algebras $\mathcal{T}_{\text {or }} \mathcal{O}_{X}\left(\mathcal{O}_{Y}, \mathcal{O}_{Y}\right)$ admits a canonical structure of a Gerstenhaber algebra such that

$$
\operatorname{Tor}_{\cdot}^{\mathcal{O}_{X}}\left(\mathcal{O}_{Y}, \mathcal{O}_{Y}\right) \simeq \wedge^{\bullet} \mathrm{N}_{X / Y}^{\vee}
$$

as Gerstenhaber algebras.

Moreover, the sheaf $\mathcal{E x t}_{\mathcal{O}_{X}}\left(\mathcal{O}_{Y}, \mathcal{O}_{Y}\right)$ admits a canonical structure of Gerstenhaber module over the Gerstenhaber algebra $\mathcal{T}_{\text {or }} \mathcal{O}_{X}\left(\mathcal{O}_{Y}, \mathcal{O}_{Y}\right)$ such that

$$
\mathcal{E x t}_{\mathcal{O}_{X}}\left(\mathcal{O}_{Y}, \mathcal{O}_{Y}\right) \simeq \wedge^{\bullet} \mathrm{N}_{X / Y}
$$

as Gerstenhaber modules, compatible in the obvious sense with the identification (8).

We denote by $H_{\bullet}^{\operatorname{vir}}\left(\mathcal{O}_{Y}, \mathcal{O}_{Y}\right)$, resp. $H_{\text {vir }}^{\bullet}\left(\mathcal{O}_{Y}, \mathcal{O}_{Y}\right)$, the cohomology of the complex $\left(\operatorname{Tor}^{\mathcal{O}_{X}}\left(\mathcal{O}_{Y}, \mathcal{O}_{Y}\right), \delta\right)$, resp. of $\left(\operatorname{Ext}_{\mathcal{O}_{X}}\left(\mathcal{O}_{Y}, \mathcal{O}_{Y}\right), \delta\right)$. Then results of Brylinski [14, Cor. 2.2.2] and Lichnerowicz [17, Thm. 2.1.4] imply:

Corollary 28. We have isomorphisms

$$
H_{\bullet}^{\operatorname{vir}}\left(\mathcal{O}_{Y}, \mathcal{O}_{Y}\right) \simeq H_{\mathrm{DR}}^{\operatorname{dim} X-\bullet}(Y) \quad \text { and } \quad H_{\mathrm{vir}}^{\bullet}\left(\mathcal{O}_{Y}, \mathcal{O}_{Y}\right) \simeq H_{\mathrm{DR}}^{\bullet}(Y)
$$

\section{References}

[1] M. J. Asensio, M. Van den Bergh, F. Van Oystaeyen, A new algebraic approach to microlocalization of filtered rings, Trans. Amer. Math. Soc. 316 (1989), no. 2, 537553.

[2] V. Baranovsky, V. Ginzburg. Gerstenhaber-Batalin-Vilkovisky structures on coisotropic intersections, Math. Res. Lett. 17 (2010), no. 2, 211-229.

[3] K. Behrend, B. Fantechi, Gerstenhaber and Batalin-Vilkovisky structures on Lagrangian intersections, in: Algebra, Arithmetic, and Geometry: In Honor of Yu. I. Manin, Vol. I, Progress in Mathematics, Vol. 269, Birkhäuser Boston, Boston, MA, 2009, pp. 1-47.

[4] G. Bellamy, Cuspidal representations of rational Cherednik algebras at $t=0$, Math. Z. 269 (2008), no. 3-4, 609-627.

[5] G. Bellamy, Rational cherednik algebras and schubert cells, arXiv:1210.3870, 2012.

[6] G. Bellamy, Symplectic reflection algebras, arXiv:1210.1239, 2012.

[7] G. Bellamy, T. Schedler, A new linear quotient of $\mathbf{C}^{4}$ admitting a symplectic resolution, Math. Z. 273 (2013), no. 3-4, 753-769.

[8] D. J. Benson, Polynomial Invariants of Finite Groups, London Mathematical Society Lecture Note Series, Vol. 190, Cambridge University Press, Cambridge, 1993.

[9] D. J. Benson, Representations and Cohomology. I, 2nd ed., Cambridge Studies in Advanced Mathematics, Vol. 30, Cambridge University Press, Cambridge, 1998.

[10] R. Bezrukavnikov, P. Etingof. Parabolic induction and restriction functors for rational Cherednik algebras, Selecta Math. (N.S.) 14 (209), no. 3-4, 397-425.

[11] A. Białynicki-Birula, Some theorems on actions of algebraic groups, Ann. of Math. (2) 98 (1973), 480-497.

[12] C. Bonnafé, R. Rouquier, Cellules de Calogero-Moser, arXiv:1302.2720, 2013. 
[13] K. A. Brown, I. G. Gordon, The ramification of centres: Lie algebras in positive characteristic and quantised enveloping algebras, Math. Z. 238 (2001), no. 4, 733779 .

[14] J-L. Brylinski, A differential complex for Poisson manifolds, J. Diff. Geom. 28 (1988), no. 1, 93-114.

[15] N. Chrissm V. Ginzburg, Representation Theory and Complex Geometry, Birkhäuser Boston, Boston, MA, 1997.

[16] S. C. Coutinho, Krull dimension of modules and involutive ideals, Proc. Amer. Math. Soc. 123 (1995), no. 6, 1647-1654.

[17] J. P. Dufour, N. T. Zung, Poisson Structures and their Normal Forms, Progress in Mathematics, Vol. 242, Birkhäuser Verlag, Basel, 2005.

[18] D. Eisenbud, Commutative Algebra, Graduate Texts in Mathematics, Vol. 150, Springer-Verlag, New York, 1995.

[19] P. Etingof, V. Ginzburg, Symplectic reflection algebras, Calogero-Moser space, and deformed Harish-Chandra homomorphism, Invent. Math. 147 (2002), no. 2, 243-348.

[20] E. Frenkel, Langlands Correspondence for Loop Groups, Cambridge Studies in Advanced Mathematics, Vol. 103, Cambridge University Press, Cambridge, 2007.

[21] E. Frenkel, C. Teleman, Self-extensions of Verma modules and differential forms on opers, Compos. Math. 142 (2006), no. 2, 477-500.

[22] B. Fu, A survey on symplectic singularities and symplectic resolutions, Ann. Math. Blaise Pascal 13 (2006), no. 2, 209-236.

[23] O. Gabber, The integrability of the characteristic variety, Amer. J. Math. 103 (1981), no. 3, 445-468.

[24] V. Ginzburg, N. Guay, E. Opdam, R. Rouquier, On the category $\mathcal{O}$ for rational Cherednik algebras, Invent. Math. 154 (2003), no. 3, 617-651.

[25] I. G. Gordon, Baby Verma modules for rational Cherednik algebras, Bull. London Math. Soc. 35 (2003), no. 3, 321-336.

[26] I. G. Gordon, Symplectic reflection algebras, in: Trends in Representation Theory of Algebras and Related Topics, EMS Ser. Congr. Rep., Eur. Math. Soc., Zürich, 2008, pp. 285-347.

[27] A. Grothendieck, Éléments de géométrie algébrique. I. Le langage des schémas, Inst. Hautes Études Sci. Publ. Math. 4 (1960), 5-228.

[28] R. Hartshorne, Algebraic Geometry, Graduate Texts in Mathematics, Vol. 52, Springer-Verlag, New York, 1977. Russian transl.: P. Хартсхорн, Алгебраическая геометрия, Мир, М., 1981.

[29] M. Kashiwara, R. Rouquier, Microlocalization of rational Cherednik algebras, Duke Math. J. 144 (2008), no. 3, 525-573.

[30] M. Kashiwara, P. Schapira, Constructibility and duality for simple holonomic modules on complex symplectic manifolds, Amer. J. Math. 130 (2008), no. 1, 207-237.

[31] J-P. Serre, Local Algebra, Springer Monographs in Mathematics, Springer-Verlag, Berlin, 2000.

[32] D. Tamarkin, Microlocal condition for non-displaceablility, arXiv:0809.1584, 2008.

[33] M. Varagnolo, E. Vasserot, Cyclotomic double affine Hecke algebras and affine parabolic category $\mathcal{O}$, Adv. Math. 225 (2010), no. 3, 1523-1588. 